\title{
APPLICATIONS OF A THEOREM OF LEVY TO BOOLEAN TERMS AND ALGEBRAS
}

BY

\author{
JONATHAN STAVI
}

ABSTRACT. The paper begins with a short proof of the Gaifman-Hales theorem and the solution of a problem of Gaifman about the depth and length of Boolean terms. The main results are refinements of the following theorem: Let $k$ be regular, $\kappa_{1}<k<\infty$. A $<k$-complete Boolean algebra on $\kappa_{0}$ generators, which are restricted by just one countably long equation, is either atomic with $<\aleph_{0}$ atoms or isomorphic to the free $<\kappa$-complete Boolean algebra on $\aleph_{0}$ generators. The main tools are a Skolem-Löwenheim type theorem of Azriel Lévy and a coding of Borel sets and Borel-measurable functions by Boolean terms.

Introduction. Lévy's theorem referred to in the title is Theorem 36 of [Lé] which states that for any cardinal $\kappa>\aleph_{0}$ the set $H(\kappa)$ of all sets of hereditary cardinality less than $K$ is a $\Sigma_{1}$-elementary substructure of the universe. Karp has shown [Ka] the usefulness of this theorem in proving $V=$ $L \Rightarrow G C H$, and since then the theorem has found diverse applications in set theory, model theory and infinitary languages (cf. $[\mathrm{Ba}],[\mathrm{Gr}]$ and $[\mathrm{Na}]$ ). Here we present applications of Lévy's theorem in the theory of Boolean terms and free Boolean algebras. Boolean terms (B.t's) were introduced under this name by Gaifman [Ga] but they can be identified with the formulas of the familiar infinitary $\left(L_{\infty}\right)$ propositional language. The standard way to obtain free Boolean algebras is to divide the collection of Boolean terms by the equivalence relation $\equiv$, where $\varphi \equiv \psi$ iff $\varphi$ and $\psi$ have the same value in all Boolean-valued models. Applications of Lévy's theorem in this area depend on the fact that the relation $\equiv$ between B.t's is $\Delta_{1}^{Z F}$ (which has also been observed by Gregorycf. [Gr, §3]).

Perhaps the best example of such an application is the short proof of the Gaifman-Hales theorem (there is a proper class of pairwise nonequivalent B.t's on $\aleph_{0}$ variables) given in $\S 1$. This proof appears also in [St, §18], but its simplicity is concealed there by the surrounding generalizations and discussion (in which it is shown that Kripke's theorem, stating that each Boolean algebra (B.a.) has a complete embedding in a countably-generated B.a., can be proved in a similar way). 
After $\S 1$, which is preliminary and illustrative, we deal with three directions in which Lévy's theorem proves useful:

(I) $\S \S 2-3$ discuss depth and length of B.t's. The main result of $\S 3$ is the solution of a problem posed by Gaifman [Ga, p. 232] about the possibility of simultaneous minimalization of depth and length in an equivalence class of Boolean terms.

(II) $\S \S 4-6$ introduce and study the notion of a confining B.t. This notion is a natural outcome of reflection about the Gaifman-Hales theorem. Consider the B.t's supported by a given set $D$ (i.e.-built up from the variables $p_{x}, x \in$ $D$ ). If $D$ is infinite then by the Gaifman-Hales theorem there is a proper class of such B.t's no two of which are equivalent. Now let $\sigma$ be a given B.t. (not necessarily supported by $D$ ), and let us replace the relation $\equiv$ by $\equiv(\sigma)$, where $\varphi \equiv \psi(\sigma)$ means: $\|\varphi\|=\|\psi\|$ whenever $\|\sigma\|=1$. Does there still exist a proper class of B.t's supported by $D$ no two of which stand in the relation $\equiv(\sigma)$ ? If there is no such class we say that $\sigma$ is confining on $D$. Let $\operatorname{Mod}_{D}(\sigma)$ be the set of (two-valued) models of the propositional language with variables $p_{x}, x \in$ $D$ (such models are regarded as points of the space $\left.D_{2}=\{x \mid x: D \rightarrow\{0,1\}\}\right)$ which are restrictions to that language of models of $\sigma$. The main result of $\S 6$ is that if $\sigma$ is of countable length, then $\sigma$ is confining on $D$ iff $\operatorname{Mod}_{D}(\sigma)$ is countable. The proof uses, besides Lévy's theorem, the connection between countable B.t's and analytic sets in the Cantor space.

(III) In this part, which contains the main results, we use the connection just mentioned together with some descriptive set theory and Lévy's theorem, to study for any regular uncountable cardinal $\kappa$, the B.a's $F / \sigma$ obtained by dividing the B.t's supported by $\omega$ of length $<_{k}$ by the equivalence relations $\equiv(\sigma)$ for various countable B.t's $\sigma$. The free $<k$-complete B.a. $F$ on $\aleph_{0}$ generators is obtained in the special case when $\sigma$ is a tautology. By the Gaifman-Hales theorem we know that $F$ is large if $k$ is (in fact, by $[\mathrm{St}],|F|=2^{k} \geqslant k$ ), and one may ask how large is $F / \sigma$ for various countable $\sigma$. This is a refinement of the question whether $\sigma$ is confining, which corresponds here to the case " $k=$ $\infty$ ". The main result is Theorem 9.5 which gives much more than an answer to this question. It states that, letting $X=\operatorname{Mod}_{\omega}(\sigma)$ ("Mod" is defined above), $F / \sigma$ is isomorphic to the B.a. of all subsets of $X$ if $X$ is countable, isomorphic to $F$ itself if $X$ is an uncountable Borel subset of the Cantor space, and not isomorphic to $F$ but each of $F / \sigma$ and $F$ has a 1-1 and an onto < $<$-complete homomorphism into the other (hence they have the same power) if $X$ is nonBorel ( $X$ is always an analytic subset of the Cantor space $\omega_{2}$ ).

Let us regard $F_{0}$, the free $\sigma$-B.a. on $\aleph_{0}$ generators, as a subalgebra of 
$F$ in the natural way. One consequence of the theorem just quoted is that many direct factors (principal ideals) of $F$ determined by elements of $F_{0}$ are isomorphic to $F$. We show that a sequence of elements of $F_{0}$ behaves, from various points of view, in the same way in $F_{0}$ and in $F(9.3,9.4,10.3)$. Also, our results show that if a $\sigma$-endomorphism of $F_{0}$ is a $1-1$ or onto then its natural extension to a $<k$-complete endomorphism of $F$ is $1-1$ or onto then its natural (see 9.3, 9.4). In $\S 10$ we show that $F$ has many automorphisms taking given elements of $F_{0}$ to others.

Actually we do not deal with $F_{0}$ in itself but identify it with the B.a. Bor of Borel subsets of $\omega_{2}$, to which it is isomorphic. And we show that the B.a. F/ $\sigma$ depends only on the analytic set $\operatorname{Mod}_{\omega}(\sigma)$ and not on the countable B.t. $\sigma$ itself, so that one can meaningfully talk of $F / X$ for any analytic set $X$. Thus we can present the results in a way which is much less syntactical and more algebraic and one can almost forget the B.t's with which one started except for the fact that almost all the interesting results and some basic definitions are justified by means of Lévy's theorem applied to syntactical predicates.

In the concluding section ( $\$ 11)$ we present some questions and indicate how the coding of Borel sets and functions by B.t's may be useful in studying analysis in ZFC models, in the spirit of Solovay [So].

Throughout the work we assume some terminology and notations concerning B.t's. For the reader's convenience these preliminaries are collected in an Appendix at the end, where the proof of $\Delta_{1}$-ness of the consequence relation $\vdash$ of Boolean logic is also sketched. Perhaps the only unusual feature is our use of inequalities between B.t's, rather than single B.t's, as hypotheses and conclusions in derivations. This device, taken from [St, §11] has some advantages, one of which is that all basic notions, up to the last paragraph of $\S 4$, generalize straightforwardly when Boolean algebras are replaced by various classes of lattices. We advise the reader to have a look at the Appendix now, and return to it whenever necessary. A less formal presentation of B.t's and the calculus of inequalities can be found in $\S 11$ of [St].

This work is a chapter of the author's Ph. D. Thesis prepared at the Hebrew University of Jerusalem under the supervision of Professor H. Gaifman, to whom the author is greatly indebted. Some of the main results have been announced in Notices Amer. Math. Soc. 18 (1971), 825, 968. Abstracts \#71T-E79, 71T-

E87. The results announced there had been worked out independently of recent applications of Lévy's theorem in the literature, with which I became acquainted much later; only the example of [Ka] guided me at that time.

For extensions of the Gaifman-Hales theorem in completely different directions we refer the reader to [St]. 
1. Lévy's theorem and its application. In the hierarchy of formulas studied in [Lé] the lowest level is that of restricted $\left(\Sigma_{0}, \Delta_{0}\right)$ formulas, which are obtained by closing atomic formulas of the basic language of set theory under the connectives and restricted quantification $(\forall u \in v, \exists u \in v) . \Sigma_{1}$ formulas are those of the form $\exists x \Phi$ where $\Phi$ is restricted. If one works in a set theory $S, \Sigma_{1}^{S}$ formulas are the formulas (possibly containing defined symbols) which are equivalent in $S$ to some $\Sigma_{1}$ formula. If $\Phi$ and its negation are both $\Sigma_{1}^{S}, \Phi$ is said to be a $\Delta_{1}^{S}$ formula. A term $\tau$ is $\Delta_{1}^{S}$ when the formula $v=\tau$ ( $v$ a new variable) is $\Delta_{1}^{S}$. We shall usually ascribe $\Delta_{1}^{\mathrm{ZF}}$-ness or $\Delta_{1}^{\mathrm{ZFC}}$-ness $(\mathrm{ZFC}=\mathrm{ZF}+$ choice) to statements written partly in English, but some natural formalization in ZF or ZFC is always assumed. For partly defined predicates and operations (e.g.-those defined only for ordinals, mappings, real numbers) the formalization is assumed to make them false or give them the value 0 outside their natural domain of applicability.

Lists of closure properties for $\Sigma_{1}^{Z F}$ and $\Delta_{1}^{Z F}$ formulas and terms can be found in [Lé] and [Ka], and the reader is assumed to be able to recognize many formulas and terms as $\Sigma_{1}^{Z F}$ or $\Delta_{1}^{Z F}$ (sometimes ZFC) even when we write them informally.

Generally speaking all "absolute" or "computable" formulas and terms (e.g.those defined by recursion of $\Theta$ ) are $\Delta_{1}^{Z F}$ (hence $\Sigma_{1}^{Z F}$ ), and existential, as well as restricted universal, quantification does not lead out of $\Sigma_{1}^{Z F}$ formulas.

Lévy's theorem is the following theorem-scheme in ZFC, in which $\Phi=$ $\Phi\left(x_{1}, \cdots, x_{n}, y_{1}, \cdots, y_{m}\right)(m \geqslant 0, n \geqslant 1)$ is any $\Sigma_{1}^{Z F C}$ formula.

$$
\begin{aligned}
\exists x_{1}, \cdots, x_{n} \Phi \Rightarrow^{*} \exists x_{1}, \cdots, & x_{n}\left[\Phi \text { and } \max \left(\left|T C\left(x_{1}\right)\right|, \cdots,\left|T C\left(x_{n}\right)\right|\right)\right. \\
& \left.\leqslant \max \left(\aleph_{0},\left|T C\left(y_{1}\right)\right|, \cdots,\left|T C\left(y_{m}\right)\right|\right)\right] .
\end{aligned}
$$

Here $T C(x)$ is the transitive closure of $x$, and $|z|$ is the power of the set z. Alternatively, putting $H(\kappa)=\{x|| T C(x) \mid<\kappa\}$ ( $\kappa$ an infinite cardinal) we may write (with $\Phi$ as above):

$$
\begin{array}{r}
\left(\kappa>\aleph_{0} \text { and } y_{1}, \cdots, y_{m} \in H(\kappa) \text { and } \exists x_{1}, \cdots, x_{n} \Phi\right) \\
\Rightarrow\left(\exists x_{1}, \cdots, x_{n} \in H(\kappa)\right) \Phi .
\end{array}
$$

The case $k=\aleph_{1}$ is most important. $H\left(\aleph_{1}\right)$ is denoted also by $H C$ and called the set of hereditarily countable sets.

Lévy's theorem is a Skolem-Löwenheim type theorem for the universe of sets, and is not hard to prove by a Skolem-Löwenheim argument (see [Lé] or [Ka]). It is very useful in extending results from the countable to the uncountable. We shall now illustrate it by proving that there is a proper class of pairwise nonequivalent B.t's supported by the countable set $\omega \times \omega$ (hence-by any given 
infinite set; this is the Gaifman-Hales theorem). In the proof we shall assume knowledge of the Appendix, particularly of the $\Sigma_{1}^{Z F}$-ness of the relation $\equiv$ between B.t's and of Definition 16.

We define the sequence $\left\langle\pi_{\alpha}^{n}\right| n\langle\omega\rangle$ of B.t's supported by $\omega \times \omega$ by recursion on $\alpha$ as follows:

$$
\pi_{\alpha}^{n}=\bigwedge_{m}\left(p_{m n} \rightarrow \underset{\beta<\alpha}{\bigvee} \pi_{\beta}^{m}\right) \wedge \bigwedge_{\beta<\alpha m} \bigvee\left(p_{m n} \wedge \pi_{\beta}^{m}\right)
$$

It is easily seen by induction on $\alpha$ that if $R \subseteq \omega \times \omega$ is any well-ordering of $\omega$ then for all $n,(\omega \times \omega, R) \vDash \pi_{\alpha}^{n}$ iff $n$ occupies the $\alpha$ th place in $R$. Now, if $\alpha$ is a countable ordinal, then there is a well-ordering $R$ of $\omega$ in which 0 occupies the $\alpha$ th place. $\pi_{\alpha}^{0}$ is true in $(\omega \times \omega, R)$ (for this $R$ ) and for all $\beta \neq \alpha, \pi_{\beta}^{0}$ is false. Therefore, the inequality $\pi_{\alpha}^{0} \leqslant \pi_{\beta}^{0}$ is false in the valuation $\left(2, I_{R}\right)$ associated with $(\omega \times \omega, R)$. Thus

$$
\alpha<\aleph_{1}, \alpha \neq \beta \Rightarrow \pi_{\alpha}^{0} \not \pi_{\beta}^{0} \text {. }
$$

Next note that " $x, y$ are distinct ordinals and $\pi_{x}^{0} \equiv \pi_{y}^{0}$ " is a $\Sigma_{1}^{Z F}$ statement, because " $\pi_{\alpha}^{n}$ " has been defined by recursion and is a $\Delta_{1}^{Z F}$-term. Thus by Lévy's theorem, if $\dot{\exists} \alpha \beta\left[\alpha \neq \beta\right.$ and $\left.\pi_{\alpha}^{0} \equiv \pi_{\beta}^{0}\right]$ then $(\exists \alpha \beta \in H C)[\alpha \neq$ $\beta$ and $\left.\pi_{\alpha}^{0} \equiv \pi_{\beta}^{0}\right]$. But $\alpha \in H C \Longleftrightarrow \alpha<\aleph_{1}$ and so

$$
\exists \alpha \beta\left[\alpha \neq \beta \text { and } \pi_{\alpha}^{0} \equiv \pi_{\beta}^{0}\right] \Rightarrow\left(\exists \alpha \beta<\aleph_{1}\right)\left[\alpha \neq \beta \text { and } \pi_{\alpha}^{0} \equiv \pi_{\beta}^{0}\right] \text {. }
$$

We have already refuted the consequent, so the antecedent is false too. Thus, for all $\alpha \neq \beta, \pi_{\alpha}^{0} \neq \pi_{\beta}^{0}$, and we have a proper class as required.

2. Measures of complexity for B.t's. The simplest measure is the depth (d)-see Appendix, Definition 5.

Since we do not regard B.t's as well-ordered sequences of symbols, we cannot speak of the length in a literal sense, but still one can seek a cardinal which will serve as a measure for the length, or size, of the B.t. For finite B.t's (i.e.B.t's $\varphi$ such that $\operatorname{Sub}(\varphi)$ is finite) there is no reason, as far as I know, to single out one particular measure of length. But for infinite B.t's the following result shows that some simple adequacy conditions determine the length completely.

2.1. Lemma. For each B.t. $\varphi$ put

$$
l_{1}(\varphi)=|\operatorname{Sub}(\varphi)|, \quad l_{2}(\varphi)=\sup \{|X| \mid \wedge X \text { or } \bigvee X \text { occurs in } \varphi\} .
$$

Then the following hold when $l$ is either $l_{1}$ or $l_{2}$ :

(1) $l(\varphi)<\aleph_{0}$ if $\varphi$ is atomic;

(2) if $\varphi=7 \psi$ or $\varphi=\bigwedge X, \psi \in X$ or $\varphi=\bigvee X, \psi \in X$, then $l(\varphi) \geqslant$ $l(\psi)$;

(3) $\varphi=\wedge X$ or $\varphi=\bigvee X \Rightarrow l(\varphi) \geqslant|X|$; 
(4) for any regular cardinal $\kappa$ :

$$
\begin{aligned}
\varphi & =\neg \psi, l(\psi)<\kappa \Rightarrow l(\varphi)<\kappa ; \\
(\varphi & =\wedge X \text { or } \varphi=\bigvee X),|X|<\kappa,(\forall \psi \in X)(l(\psi)<\kappa) \Rightarrow l(\varphi)<\kappa .
\end{aligned}
$$

If $l$ is any function into cardinals whose domain is a set (or class) $T$ of B.t's closed under subterms, and (1)-(4) holds for all $\varphi, \psi \in T$ then for all $\varphi \in T$ we have

(5) $\varphi$ is finite $\Rightarrow l(\varphi)<\aleph_{0} ; \varphi$ is infinite $\Rightarrow l(\varphi)=l_{1}(\varphi)=l_{2}(\varphi)$.

In particular, $l_{1}(\varphi)=l_{2}(\varphi) \geqslant \aleph_{0}$ for all infinite $\varphi$.

Proof (outlined). It is easy to see that $l_{1}$ satisfies (1)-(4). Next, let $l$ satisfy (1)-(4), and let $k$ be regular. Show by induction of $\varphi \in \operatorname{dom}(l)$ that $l_{2}(\varphi)<k \Rightarrow l(\varphi)<k$ (use (1), (4)). Hence, $\varphi$ is finite $\Rightarrow l(\varphi)<\aleph_{0}$, and $-\varphi$ is infinite $\Rightarrow l(\varphi)<\left(l_{2}(\varphi)\right)^{+}$. But by (3) $l(\varphi) \geqslant l_{2}(\varphi)$, so if $\varphi$ is infinite $l(\varphi)$ $=l_{2}(\varphi)$.

Since we are mainly interested in infinite B.t's we shall from now on, refer to $|\operatorname{Sub}(\varphi)|$ as the length of $\varphi$ and say that $\varphi$ is finite (countable) when $\operatorname{Sub}(\varphi)$ is finite (countable).

Sometimes the following measure is considered: $k(\varphi)=$ smallest infinite cardinal $k$ such that $|X|<\kappa$ whenever $\wedge X$ or $\vee X$ occurs in $\varphi$. Clearly $k(\varphi)=\aleph_{\sigma}$ iff $\varphi$ is finite, and if $k(\varphi)=\lambda^{+}$then $|\operatorname{Sub}(\varphi)|=l_{2}(\varphi)=\lambda$, and if $k(\varphi)$ is a limit cardinal then $|\operatorname{Sub}(\varphi)|=l_{2}(\varphi)=k(\varphi)$. If $\kappa$ is regular then $k(\varphi) \leqslant k \Rightarrow|\operatorname{Sub}(\varphi)|<k$ (induction on $\varphi$ ), and therefore $k(\varphi)$ is never a limit regular cardinal. Thus $|\operatorname{Sub}(\varphi)|$ determines $k(\varphi)$ uniquely except when $|\operatorname{Sub}(\varphi)|$ is singular. The following lemma will be useful for this case.

2.2. LemmA. Let $\alpha$ be a singular cardinal, $\varphi$ a B.t. such that $k(\varphi) \leqslant \kappa^{+}$. Then there is a B.t. $\varphi^{*}$ such that $\varphi^{*} \equiv \varphi, \operatorname{Supp}\left(\varphi^{*}\right)=\operatorname{Supp}(\varphi), k\left(\varphi^{*}\right) \leqslant \kappa$ and $d\left(\varphi^{*}\right) \leqslant 2 \cdot d(\varphi)$.

(Except for the bound on $d\left(\varphi^{*}\right)$ the lemma is well known and quite easy.)

Proof. Since $k$ is singular we can choose $a$ $\left\langle\alpha_{i}\right| i\langle\lambda\rangle$ cofinal in $\kappa$. Now proceed by induction on $\varphi$. If $\varphi$ is atomic take $\varphi^{*}=\varphi$. If $\varphi=\neg \psi$ and $\psi^{*}$ corresponds to $\psi$, take $\varphi^{*}=\neg \psi^{*}$ and check that it works. If $\varphi=\wedge X$ and for each $\psi \in X \psi^{*}$ corresponds to $\psi$ and $|X|<\kappa$ take $\varphi^{*}=\bigwedge_{\psi \in X} \psi^{*}$ and check that it works. Now suppose $\varphi=$ $\bigwedge_{\gamma<\kappa} \psi_{\gamma}$, and $\psi_{\gamma}^{*}$ corresponds to $\psi_{\gamma}$ for all $\gamma<\kappa$. One is tempted to try $\varphi^{*}=$ $\bigwedge_{i<\lambda} \Lambda_{\gamma<\alpha_{i}} \psi_{\gamma}^{*}$. This choice is good when $\max _{\gamma<\kappa} d\left(\psi_{\gamma}\right)$ exists, i.e. when $d(\varphi)$ is a successor ordinal. To handle the general case put $\nu=\operatorname{cf}(d(\varphi))$, so that $\nu$ is either 1 or a regular cardinal, and let $\left\langle\delta_{j}\right| j\langle\nu\rangle$ be such that $d(\varphi)=$ 
$\operatorname{Sup}_{j<\nu} \delta_{j}$. Since $d(\varphi)=\operatorname{Sup}_{\gamma<\kappa} d\left(\psi_{\gamma}\right)$ it is clear that $\nu \leqslant \kappa$, but $\kappa$ is singular so $\nu<\kappa$. For $i<\lambda, j<\nu$ let $A_{i j}=\left\{\gamma \mid \gamma<\alpha_{i}, d\left(\psi_{\gamma}\right) \leqslant \delta_{j}\right\}$. Then $\left|A_{i j}\right| \leqslant$ $\alpha_{i}<\kappa$ and $\kappa=\bigcup_{i<\lambda, j<\nu} A_{i j}$. Take $\varphi^{*}=\bigwedge_{i<\lambda, j<\nu}\left(\bigwedge_{\gamma \in A_{i j}} \psi_{\gamma}^{*}\right)$. The various conditions on $\varphi^{*}$ are easily checked, so we shall only estimate $d\left(\varphi^{*}\right)$. By the choice of $\psi_{\gamma}^{*}, d\left(\psi_{\gamma}^{*}\right) \leqslant 2 \cdot d\left(\psi_{\gamma}\right)$, hence $\gamma \in A_{i j} \Rightarrow d\left(\psi_{\gamma}^{*}\right) \leqslant 2 \cdot \delta_{j}$. Thus $d\left(\bigwedge_{\gamma \in A_{i j}} \psi_{\gamma}^{*}\right) \leqslant 2 \cdot \delta_{j}+1<2 \cdot d(\varphi)$ (because $\delta<d(\varphi) \Rightarrow 2 \cdot \delta+2 \leqslant 2 \cdot d(\varphi)$ ), hence $d\left(\varphi^{*}\right) \leqslant 2 \cdot d(\varphi)$. The treatment of $\varphi=\bigvee X$ is the same, so the proof is complete.

We shall need the following connection between depth and length.

2.3. Lemma. For every B.t. $\varphi$ :

$$
d(\varphi)=\{d(\psi) \mid \psi \in \operatorname{Sub}(\varphi), \psi \neq \varphi\}
$$

and hence $|d(\varphi)|+1 \leqslant|\operatorname{Sub}(\varphi)|$.

The proof by induction is left to the reader.

3. Simultaneous minimalization of depth and length. This section has evolved from a problem posed by Gaifman ([Ga, p. 232, last paragraph], the question concerning $B T^{\alpha}$ and $B T_{\beta}$ ). Ignoring some accidental features of his formulation (to which we return below), the problem is whether among the B.t's equivalent to a given one, there is one for which both the depth and length are minimal. Since we have no natural measure of length for finite B.t's, we shall, in a sense, ignore them or regard them all of minimal length. We generalize the problem by considering equivalence modulo any set $\Gamma_{0}$ of inequalities rather than modulo $\varnothing$ only, and consider also the support, or its power, as an additional measure which one may ask to minimalize.

It is convenient to extend the notation "Sub" to inequalities by letting $\operatorname{Sub}(\varphi \leqslant \psi)=\{\varphi \leqslant \psi\} \cup \operatorname{Sub}(\varphi) \cup \operatorname{Sub}(\psi)$, and to put $\operatorname{Sub}(K)=\bigcup_{a \in K} \operatorname{Sub}(a)$ when $K$ is any set of B.t's and inequalities.

From now on let $\Gamma_{0}$ be a fixed set of inequalities and $\kappa_{0}=$ $\max \left(\aleph_{0},\left|\operatorname{Sub}\left(\Gamma_{0}\right)\right|\right)$. For any B.t. $\varphi$ let us denote $l_{0}(\varphi)=\max \left(\kappa_{0},|\operatorname{Sub}(\varphi)|\right)$.

3.1. Lemma. If $\varphi \equiv \psi\left(\Gamma_{0}\right)$ then there is a B.t. $\chi$ such that $\varphi \equiv \chi\left(\Gamma_{0}\right)$, $l_{0}(\chi) \leqslant l_{0}(\varphi)$ and $d(\chi) \leqslant d(\psi)$.

Proof. Let $\varphi, \psi$ be as above, $l_{0}(\varphi)=\lambda$. Thus $\lambda \geqslant|\operatorname{Sub}(\varphi)| \geqslant$ $|\operatorname{Supp}(\varphi)|$ and $\lambda \geqslant \kappa_{0} \geqslant\left|\operatorname{Supp}\left(\Gamma_{0}\right)\right|$ where, naturally, $\operatorname{Supp}\left(\Gamma_{0}\right)=\left\{x \mid p_{x} \in\right.$ $\left.\operatorname{Sub}\left(\Gamma_{0}\right)\right\}$. Therefore, by renaming the atomic B.t's occurring in $\varphi, \psi$ and $\Gamma_{0}$, we may assume that $\operatorname{Supp}(\varphi) \cup \operatorname{Supp}\left(\Gamma_{0}\right) \subseteq \lambda$. Since $\varphi$ is supported by $\lambda$ and of length $\leqslant \lambda$ it is easy to see (by induction on $\varphi$ ) that $\varphi \in H\left(\lambda^{+}\right)$. In the same way, each inequality in $\Gamma_{0}$, as well as $\Gamma_{0}$ itself, is in $H\left(\lambda^{+}\right)$. Denote 
$\beta=d(\psi)$. By $2.3,|d(\varphi)| \leqslant|\operatorname{Sub}(\varphi)| \leqslant \lambda$. But if $d(\varphi) \leqslant d(\psi)$ then $\chi=\varphi$ satisfies the requirements, so we may assume $\beta<d(\varphi)$, hence $|\beta| \leqslant \lambda, \beta \in$ $H\left(\lambda^{+}\right)$. Let $\Phi\left(x, \Gamma_{0}, \varphi, \beta\right)$ be the set-theoretical statement: $x$ is a B.t., $\varphi \equiv$ $x\left(\Gamma_{0}\right)$ and $d(x)=\beta$. Clearly $\Phi$ is a $\Sigma_{1}^{Z F}$ statement, satisfied by $x=\psi$. Thus, by Lévy's theorem and the fact that $\Gamma_{0}, \varphi, \beta \in H\left(\lambda^{+}\right)$, we conclude that a B.t. $\chi \in H\left(\lambda^{+}\right)$exists such that $\varphi \equiv \chi\left(\Gamma_{0}\right), d(\chi)=\beta$. But

$$
\chi \in H\left(\lambda^{+}\right) \Rightarrow|T C(\chi)| \leqslant \lambda \Rightarrow|\operatorname{Sub}(\chi)| \leqslant \lambda \Rightarrow l_{0}(\chi) \leqslant \lambda .
$$

and thus $l_{0}(\chi) \leqslant l_{0}(\varphi)$, which completes the proof.

3.2. TheOREM. For each B.t. $\varphi$ there exists a $\varphi^{*} \equiv \varphi\left(\Gamma_{0}\right)$ such that $\operatorname{Supp}\left(\varphi^{*}\right) \subseteq \operatorname{Supp}(\varphi) \cup \operatorname{Supp}\left(\Gamma_{0}\right)$ and for all $\psi$, if $\psi \equiv \varphi\left(\Gamma_{0}\right)$ then $d\left(\varphi^{*}\right) \leqslant$ $d(\psi), l_{0}\left(\varphi^{*}\right) \leqslant l_{0}(\psi), s_{0}\left(\varphi^{*}\right) \leqslant s_{0}(\psi)$. Here $l_{0}(\psi)=\max \left(\kappa_{0},|\operatorname{Sub}(\psi)|\right), s_{0}(\psi)$ $=\max \left(\kappa_{0},|\operatorname{Supp}(\psi)|\right)$.

Proof. Among the B.t's equivalent to $\varphi$ modulo $\Gamma_{0}$ choose three $\psi_{1}, \psi_{2}, \psi_{3}$ for which the measures $d, l_{0}, s_{0}$ respectively are minimal. Applying 3.1 to $\psi_{2}$ and $\psi_{1}$ we get a $\psi_{4} \equiv \varphi\left(\Gamma_{0}\right)$ such that $d\left(\psi_{4}\right) \leqslant d\left(\psi_{1}\right)$ and $l_{0}\left(\psi_{4}\right) \leqslant l_{0}\left(\psi_{2}\right)$. Now replace in $\psi_{4}$ every atom $p_{x}$ which occurs neither in $\Gamma_{0}$ nor in $\psi_{3}$ by $\Lambda \varnothing$. The result is a B.t. $\psi_{5}$, and $\psi_{4} \equiv \psi_{3}\left(\Gamma_{0}\right) \Rightarrow \psi_{5} \equiv$ $\psi_{3}\left(\Gamma_{0}\right)$. It is also easy to see that $d\left(\psi_{5}\right)=d\left(\psi_{4}\right)$ and that every subterm of $\psi_{5}$ is obtained by the above substitution from some subterm of $\psi_{4}$, so that $l_{0}\left(\psi_{5}\right) \leqslant l_{0}\left(\psi_{4}\right)$. Clearly $\operatorname{Supp}\left(\psi_{5}\right) \subseteq \operatorname{Supp}\left(\psi_{3}\right) \cup \operatorname{Supp}\left(\Gamma_{0}\right)$. Finally let $\varphi^{*}$ result from $\psi_{5}$ by replacing each atom $p_{x}$ not occurring in $\Gamma_{0}$ nor in $\varphi$ by $\wedge \varnothing$. Since $\psi_{5} \equiv \varphi\left(\Gamma_{0}\right), \varphi^{*} \equiv \varphi\left(\Gamma_{0}\right)$. Also, $d\left(\varphi^{*}\right)=d\left(\psi_{5}\right)=d\left(\psi_{4}\right) \leqslant d\left(\psi_{1}\right)$, $l_{0}\left(\varphi^{*}\right) \leqslant l_{0}\left(\psi_{5}\right) \leqslant l_{0}\left(\psi_{4}\right) \leqslant l_{0}\left(\psi_{2}\right), \operatorname{Supp}\left(\varphi^{*}\right) \subseteq \operatorname{Supp}(\varphi) \cup \operatorname{Supp}\left(\Gamma_{0}\right)$ and $\operatorname{Supp}\left(\varphi^{*}\right) \subseteq \operatorname{Supp}\left(\psi_{5}\right) \subseteq \operatorname{Supp}\left(\psi_{3}\right) \cup \operatorname{Supp}\left(\Gamma_{0}\right) ;$ hence $s_{0}\left(\varphi^{*}\right) \leqslant s_{0}\left(\psi_{3}\right)$. Thus $d, l_{0}, s_{0}$ all get their minimum at $\varphi^{*}$, which is the desired B.t.

Theorem 3.2 is unsatisfactory for the case $\left|\operatorname{Sub}\left(\Gamma_{0}\right)\right|<\aleph_{0}$, because then $\kappa_{0}=\aleph_{0}$ and we have no more information than we would have if we only knew $\left|\operatorname{Sub}\left(\Gamma_{0}\right)\right| \leqslant \aleph_{0}$. We have the following result for this case.

3.3. Suppose $\operatorname{Sub}\left(\Gamma_{0}\right)$ is finite. For every B.t. $\varphi$ there is a B.t. $\varphi^{*} \equiv$ $\varphi\left(\Gamma_{0}\right)$ such that $\operatorname{Supp}\left(\varphi^{*}\right) \subseteq \operatorname{Supp}(\varphi) \cup \operatorname{Supp}\left(\Gamma_{0}\right)$ and whenever $\varphi \equiv \psi\left(\Gamma_{0}\right)$ we have

$$
d\left(\varphi^{*}\right) \leqslant d(\psi), \quad\left|\operatorname{Sub}\left(\varphi^{*}\right)\right| \leqslant|\operatorname{Sub}(\psi)|+n \quad \text { for some } n<\omega,
$$

and

$$
\left|\operatorname{Supp}\left(\varphi^{*}\right) \sim \operatorname{Supp}\left(\Gamma_{0}\right)\right| \leqslant\left|\operatorname{Supp}(\psi) \sim \operatorname{Supp}\left(\Gamma_{0}\right)\right|
$$

(hence $\left|\operatorname{Supp}\left(\varphi^{*}\right)\right| \leqslant|\operatorname{Supp}(\psi)|$ in case $\Gamma_{0}=\varnothing$ ).

PROOF. For the purposes of this proof define, for each B.t., $\psi: l_{0}(\psi)=0$ 
if $\psi$ is finite, $=|\operatorname{Sub}(\psi)|$ otherwise; $s_{0}(\psi)=\left|\operatorname{Supp}(\psi) \sim \operatorname{Supp}\left(\Gamma_{0}\right)\right|$. Among the B.t's equivalent to $\varphi$ modulo $\Gamma_{0}$ choose three $\psi_{1}, \psi_{2}, \psi_{3}$ for which $d$, $l_{0}, s_{0}$ respectively are minimal. Our first object is to find some $\psi_{4} \equiv \varphi\left(\Gamma_{0}\right)$ with $d\left(\psi_{4}\right) \leqslant d\left(\psi_{1}\right), l_{0}\left(\psi_{4}\right) \leqslant l_{0}\left(\psi_{2}\right)$. If $\psi_{2}$ is infinite this amounts to finding $\psi_{4}$ with $d\left(\psi_{4}\right) \leqslant d\left(\psi_{1}\right), \max \left(\aleph_{0},\left|\operatorname{Sub}\left(\psi_{4}\right)\right|\right) \leqslant \max \left(\aleph_{0},\left|\operatorname{Sub}\left(\psi_{2}\right)\right|\right)$, which can be done by 3.1. If $\psi_{2}$ is finite but $d\left(\psi_{1}\right)$ is infinite we can take $\psi_{4}=\psi_{2}$. The remaining case is that $d\left(\psi_{1}\right)<\omega$ and $\psi_{2}$ is finite. Then let $\psi_{4}$ be the result of substituting in $\psi_{1} \Lambda \varnothing$ for each occurrence of an atomic B.t. that occurs neither in $\psi_{2}$ nor in $\Gamma_{0}$. Clearly $d\left(\psi_{4}\right)=d\left(\psi_{1}\right)<\omega$ and $\operatorname{Supp}\left(\psi_{4}\right) \subseteq \operatorname{Supp}\left(\psi_{2}\right) \cup \operatorname{Supp}\left(\Gamma_{0}\right)$. Since $\psi_{1} \equiv \psi_{2}\left(\Gamma_{0}\right), \psi_{4} \equiv \psi_{2}\left(\Gamma_{0}\right)$. It remains only to check that $l_{0}\left(\psi_{4}\right) \leqslant l_{0}\left(\psi_{2}\right)=0$, i.e., that $\psi_{4}$ is finite. But $d\left(\psi_{4}\right)<\omega$ and $\left|\operatorname{Supp}\left(\psi_{4}\right)\right| \leqslant\left|\operatorname{Supp}\left(\psi_{2}\right) \cup \operatorname{Supp}\left(\Gamma_{0}\right)\right| \leqslant\left|\operatorname{Sub}\left(\psi_{2}\right) \cup \operatorname{Sub}\left(\Gamma_{0}\right)\right|$ $<\aleph_{0}$, so the finiteness of $\psi_{4}$ follows from the

\section{Lemma. A B.t. of finite support and finite depth is finite.}

The lemma follows directly from the observation that if $A$ is a finite set, $n<\omega$ then there are only finitely many B.t's supported by $A$ of depth $\leqslant n$ (proof by induction on $n$ ).

We have thus found $\psi_{4}$ as desired. Next define $\psi_{5}$ and $\varphi^{*}$ exactly as in the proof of 3.2. Then $d\left(\varphi^{*}\right)=d\left(\psi_{5}\right)=d\left(\psi_{4}\right) \leqslant d\left(\psi_{1}\right)$. Also $\left|\operatorname{Sub}\left(\varphi^{*}\right)\right| \leqslant$ $\left|\operatorname{Sub}\left(\psi_{5}\right)\right| \leqslant\left|\operatorname{Sub}\left(\psi_{4}\right)\right|$ because every subterm of $\psi_{5}$ (of $\varphi^{*}$ ) results by a certain substitution from a subterm of $\psi_{3}$ (of $\psi_{5}$ resp.). Therefore $l_{0}\left(\varphi^{*}\right) \leqslant$ $l_{0}\left(\psi_{4}\right) \leqslant l_{0}\left(\psi_{2}\right)$. The definitions of $\psi_{5}$ and $\varphi^{*}$ imply that

$$
\begin{aligned}
\operatorname{Supp}\left(\varphi^{*}\right) \sim \operatorname{Supp}\left(\Gamma_{0}\right) & \subseteq \operatorname{Supp}\left(\psi_{5}\right) \sim \operatorname{Supp}\left(\Gamma_{0}\right) \\
& \subseteq \operatorname{Supp}\left(\psi_{3}\right) \sim \operatorname{Supp}\left(\Gamma_{0}\right),
\end{aligned}
$$

hence $s_{0}\left(\varphi^{*}\right) \leqslant s_{0}\left(\psi_{3}\right)$. Also $\operatorname{Supp}\left(\varphi^{*}\right) \subseteq \operatorname{Supp}(\varphi) \cup \operatorname{Supp}\left(\Gamma_{0}\right)$ and $\varphi^{*} \equiv$ $\varphi\left(\Gamma_{0}\right)$. Thus $\varphi^{*}$ has all the properties listed in 3.3 (note that " $\exists n\left(\left|\operatorname{Sub}\left(\varphi^{*}\right)\right| \leqslant\right.$ $|\operatorname{Sub}(\psi)|+n) "$ is merely another way of saying that $\left.l_{0}\left(\varphi^{*}\right) \leqslant l_{0}(\psi)\right)$.

REMARK. When $\Gamma_{0}=\varnothing$ we see that we can minimize simultaneously, in each equivalence class, $d(\psi), l_{0}(\psi)$ and $|\operatorname{Supp}(\psi)|$ where $l_{0}(\psi)=0$ if $\psi$ is finite, $|\operatorname{Sub}(\psi)|$ otherwise. However $\operatorname{Supp}(\psi)$ as a set cannot in general be minimized. For example take $\varphi=\bigwedge_{n} \bigvee_{m \leqslant n} p_{m}(m, n$ vary on $\omega)$. Then the possible supports of B.t's $\psi \equiv \varphi$ are easily seen to be just the sets $A$ such that $\omega \sim A$ is finite, and there is no minimal such sets.

Although our results for the case $\Gamma=\varnothing$ essentially give what Gaifman was looking for in formulating his problem [Ga, p. 232] they do not solve it in the form actually posed. In our notation (recall the definition of $k(\varphi)$ from $\S 2$ ) 
the original problem can be stated thus. Let $\delta$ be an ordinal, $B T^{*}=\{\varphi \mid \varphi$ is a B.t. supported by $\delta, k(\varphi) \leqslant \kappa\}, B T_{0}=\left\{p_{x} \mid x \in \delta\right\}$, and for $\beta>0, B T_{\beta}=$ $\{\varphi \mid \varphi$ is a B.t. supported by $\delta, d(\varphi) \leqslant \beta\}$. Suppose $\varphi \equiv \psi, \varphi \in B T^{\kappa}, \psi \in B T_{\beta}$. Is there some $\chi \equiv \varphi$ such that $\chi \in B T^{k} \cap B T_{\beta}$ ?

Since for $\beta=0$ the answer is trivially positive (take $\chi=\psi$ ), a positive answer for regular $\kappa$ is contained in the following result, which also gives some information about what happens when $\kappa$ is singular.

3.4. Theorem. Let $\varphi, \psi$ be equivalent B.t's. Then there is a B.t. $\chi$ such that

$$
\varphi \equiv \chi \equiv \psi, \quad \operatorname{Supp}(\chi) \subseteq \operatorname{Supp}(\varphi) \cap \operatorname{Supp}(\psi), \quad k(\chi) \leqslant k(\varphi)
$$

and

$$
d(\chi) \leqslant\left\{\begin{array}{l}
d(\psi) \text { if } k(\varphi) \text { is regular, } \\
2 \cdot d(\psi) \text { if } k(\varphi) \text { is singular. }
\end{array}\right.
$$

Proof. By 3.3 for $\Gamma_{0}=\varnothing$ a B.t. $\varphi^{*} \equiv \varphi$ can be found such that $\operatorname{Supp}\left(\varphi^{*}\right) \subseteq \operatorname{Supp}(\varphi),\left|\operatorname{Sub}\left(\varphi^{*}\right)\right| \leqslant|\operatorname{Sub}(\varphi)|+n$ for some $n$ and $d\left(\varphi^{*}\right) \leqslant d(\psi)$. By the now familiar substitution of $\Lambda \varnothing$ for certain atomic B.t's we may assume that $\operatorname{Supp}\left(\varphi^{*}\right) \subseteq \operatorname{Supp}(\psi)$, i.e., $\operatorname{Supp}\left(\varphi^{*}\right) \subseteq \operatorname{Supp}(\varphi) \cap \operatorname{Supp}(\psi)$. Denote $\kappa=$ $k(\varphi)$. If $k$ is regular then, as noted in $\S 2$, $|\operatorname{Sub}(\varphi)|<\kappa$, and so $\left|\operatorname{Sub}\left(\varphi^{*}\right)\right|<$ $\kappa, k\left(\varphi^{*}\right) \leqslant \kappa$. For this case $\varphi^{*}$ is the desired $\chi$ of the theorem. If $\kappa$ is singular then $|\operatorname{Sub}(\varphi)| \leqslant k$; hence $\left|\operatorname{Sub}\left(\varphi^{*}\right)\right| \leqslant \kappa, k\left(\varphi^{*}\right) \leqslant \kappa^{+}$. Applying 2.2 to $\varphi^{*}$ (there called $\varphi$ ) we get a $\chi \equiv \varphi^{*}(\equiv \varphi)$ with the same support such that $k(\chi) \leqslant \kappa=k(\varphi)$ and $d(\chi) \leqslant 2 \cdot d\left(\varphi^{*}\right) \leqslant 2 \cdot d(\psi) . \chi$ is the desired B.t.

To see that if $\kappa$ is singular one cannot always find $\chi$ as in 3.4 with $d(\chi) \leqslant d(\psi)$ consider the following example in which $\left\langle\alpha_{i}\right| i\langle\lambda\rangle$ is any sequence cofinal in $\kappa$ and $\lambda<\kappa$. Take $\varphi=\bigwedge_{i<\lambda} \bigwedge_{\gamma<\alpha_{i}} p_{\gamma}, \psi=\bigwedge_{\gamma<\kappa} p_{\gamma}$. Then $k(\varphi)=\kappa, d(\psi)=1$, but it is easy to see that if $\chi \equiv \psi$ and $d(\chi) \leqslant 1$ then $k(\chi)>\kappa$. Thus, simultaneous minimization of $d$ and $k$ is not always possible.

4. Confining sets of inequalities. Let $D$ be a set, $\Gamma$ a set of inequalities (not necessarily supported by $D$ ). We shall say that $\Gamma$ is confining on $D$ when it entails so many equivalences between B.t's supported by $D$ that it confines them, up to equivalence, within a set. More precisely $\Gamma$ is confining on $D$ when there is a set $U$ of B.t's such that $(\forall \varphi$ supported by $D)(\exists \psi \in U)(\varphi \equiv$ $\psi(\Gamma)$ ). This notion is interesting because there exist nonconfining sets. In fact the Gaifman-Hales theorem is (in ZFC) just the assertion that if $D$ is infinite $\varnothing$ is not confining on $D$. (If $|D|=n<\omega$ it is easy to see that every $\Gamma$ is confining on $D$, as $\equiv(\Gamma)$ has at most $2^{2^{n}}$ equivalence classes on the B.t's supported by $D$.) 
When $\Gamma$ is confining on $D$ we may form a complete B.a. whose members are in one-to-one correspondence with the equivalence classes of $\equiv(\Gamma)$ on B.t's supported by $D$. This can be compared with the algebra of Boolean polynomials (which is a proper class) defined by Gaifman $[\mathrm{Ga}, \S 0]$. The following theorem and its proof characterize the desired complete B.a. when $\Gamma$ is confining.

4.1. TheOREM. For any set $D$ and set of inequalities $\Gamma$ the following conditions are equivalent:

(1) $\Gamma$ is confining on $D$;

(2) there is a set $T$ of B.t's supported by $D$ which is closed under 7 , $\Lambda, \vee$ and subterms, and contains $p_{x}$ for each $x \in D$ and satisfies: $(\forall X \subseteq T)$ $(\exists \varphi \in T)[\varphi \equiv \bigvee X(\Gamma)]$;

(3) there is a valuation $(B, I)$ such that

(i) $B$ is complete, $I: D \rightarrow B$;

(ii) $(\forall b \in B)(\exists \varphi$ supported by $D)\left[b=\|\varphi\|_{B, I}\right]$;

(iii) $(\forall \varphi, \psi$ supported by $D)\left[\|\varphi\|_{B, I}=\|\psi\|_{B, I} \Longleftrightarrow \varphi \equiv \psi(\Gamma)\right]$.

Moreover, when $T$ is as in (2) then letting $B=T / \Gamma \vdash, I=\left\langle\left[p_{x}\right] \mid x \in D\right\rangle$, $(B, I)$ satisfied (3). Also a valuation satisfying (3) is unique up to isomorphism.

Proof. (1) $\Rightarrow(2)$. Assuming (1) choose $T_{0}$ as a set of B.t's supported by $D$ such that $(\forall \varphi$ supported by $D)\left(\exists \psi \in T_{0}\right)[\varphi \equiv \psi(\Gamma)]$. Then let $T$ be the closure of $T_{0} \cup\left\{p_{x} \mid x \in D\right\}$ under $\neg, \wedge, \vee$ and subterms.

(2) $\Rightarrow$ (3). Let $T$ be as in (2), $B=T / \Gamma \vdash, I=\left\langle\left[p_{x}\right] \mid x \in D\right\rangle$. Thus $B=\{[\varphi] \mid \varphi \in T\}$ and $[\varphi]=\|\varphi\|_{B, I}$ for all $\varphi \in T$. So (3)(ii) holds, and the completeness of $B$ is due to the fact that $(\forall X \subseteq T)(\exists \varphi \in T)[\varphi \equiv \bigvee X(\Gamma)]$. Since $B$ is complete every B.t. supported by $D$ is defined in $(B, D)$. For any $b \in B$ put $\chi(b)=\bigvee\{\psi \in T \mid[\psi]=b\}$. It is easy to see that

$$
b=\urcorner^{B} b_{1} \Rightarrow\left(\chi(b) \equiv \neg \chi\left(b_{1}\right)\right)(\Gamma), \quad b=\bigvee^{B} A \Rightarrow\left(\chi(b) \equiv \bigvee_{a \in A} \chi(a)\right)(\Gamma)
$$

and similarly for $\Lambda$. Hence, by induction on $\varphi$ we can show that if $\|\varphi\|_{B, I}=$ $b$ then $(\varphi \equiv \chi(b))(\Gamma)$. It follows that if $\|\varphi\|_{B, I}=\|\psi\|_{B, I}$ then $\varphi \equiv \psi(\Gamma)$. Conversely, if $\varphi \equiv \psi(\Gamma), b_{1}=\|\varphi\|_{B, I}, b_{2}=\|\psi\|_{B, I}$ then $\left(\chi\left(b_{1}\right) \equiv \chi\left(b_{2}\right)\right)(\Gamma)$, hence choosing $\psi_{1}$ and $\psi_{2}$ in $T$ so that $b_{1}=\left[\psi_{1}\right], b_{2}=\left[\psi_{2}\right]$ we get $\psi_{1} \equiv \psi_{2}(\Gamma)$ and so $b_{1}=\left[\psi_{1}\right]=\left[\psi_{2}\right]=b_{2}$, i.e., $\|\varphi\|_{B, I}=\|\psi\|_{B, I}$. The proof of (3), assuming (2), is complete. The proof that $(B, I)$ in (3) is unique up to isomorphism and that $(3) \Rightarrow(1)$ is easy.

Let us fix a standard procedure for getting $(B, I)$ as in (3) when $\Gamma$ is confining on $D$. For example, for each ordinal $\alpha$ let $T_{\alpha}=\{\varphi \mid \varphi$ is supported by $D, d(\varphi)<\alpha\}$, and let $\alpha_{0}$ be the smallest limit $\alpha$ such that $(\forall \varphi$ supported by $D)\left(\exists \psi \in T_{\alpha}\right)(\varphi \equiv \psi(\Gamma))$. Take $T=T_{\alpha_{0}}$ and then $4.1(2)$ holds, and 
$(B, I)$ can be formed as described in 4.1. [Alternatively, we could follow Gaifman's construction of Boolean polynomials with $\equiv(\Gamma)$ in place of $\equiv$.] The resulting B.a. $B$, which is described by $4.1(3)$ up to isomorphism, may appropriately be called the algebra of $\Gamma$-Boolean polynomials over $D$, and denoted by $B P_{D}(\Gamma)$.

The following result shows that every complete B.a. can be represented as an algebra of Boolean polynomials.

4.2. THEOREM. If $B$ is a complete, $\leqslant|D|$-generated B.a., then there is a set $\Gamma$ of inequalities supported by $D$ and confining on $D$ such that $B \cong$ $B P_{D}(\Gamma)$.

Proof. Let $B$ be complete and $\leqslant|D|$-generated. Choose $I: D \rightarrow B$ so that range $(I)$ generates $B$. Then $(\forall b \in B) \exists \varphi\left(\|\varphi\|_{B, I}=b\right)$ and so there is a set $T$ of B.t's, closed under $ᄀ, \wedge, \vee$ and subterms, such that $D=\left\{x \mid p_{x} \in\right.$ $T\}$ and $(\forall b \in B)(\exists \varphi \in T)\left(\|\varphi\|_{B, I}=b\right)$. Let

$$
\begin{aligned}
& \Gamma_{1}=\left\{\varphi \leqslant \psi \mid \varphi, \psi \in T,\|\varphi\|_{B, I} \leqslant\|\psi\|_{B, I}\right\}, \\
& \Gamma_{2}=\left\{\varphi \leqslant \vee X \mid \varphi \in T, X \subseteq T,\|\varphi\|_{B, I}=\bigvee_{\psi \in X}^{B}\|\psi\|_{B, I}\right\}, \quad \Gamma=\Gamma_{1} \cup \Gamma_{2} .
\end{aligned}
$$

Let the reader check that $T$ and $\Gamma$ satisfy $4.1(2)$ and that $[\varphi] \mapsto\|\varphi\|_{B, I}$ $(\varphi \in T)$ is an isomorphism between $T / \Gamma \vdash$ and $B$. But by 4.1 and the definitions, $T / \Gamma \vdash \cong B P_{D}(\Gamma)$, so $B \cong B P_{D}(\Gamma)$.

4.1 and 4.2 are only the first obvious steps in a systematic study of confining sets. We shall not pursue any more generalities now, and instead we shall turn our attention to the determination whether a given $\Gamma$ is confining or not on a given set $D$. When $\Gamma$ is a finite set of inequalities between finite B.t's (in short $|\operatorname{Sub}(\Gamma)|<\aleph_{0}$ ), it is obvious (using the Gaifman-Hales theorem) that $\Gamma$ is confining on $D$ iff $D$ is finite or $\Gamma$ is inconsistent. We therefore pass to the next simplest situation $-|\operatorname{Sub}(\Gamma)| \leqslant \aleph_{0}$ (the case of a countable set of inequalities between finite B.t's is not appreciably simpler and is included in the above). Finding a necessary and sufficient condition for $\Gamma$ being confining on $D$ when $|\operatorname{Sub}(\Gamma)| \leqslant \aleph_{0}$ is one of the main tasks of the following sections, but various related results concerning free B.a's will also be obtained.

For the whole discussion, it will be essential to know that each set $\Gamma$ of inequalities is equivalent to the single inequality $\wedge \varnothing \leqslant \sigma$ where $\sigma=\{\varphi \rightarrow$ $\psi \mid(\varphi \leqslant \psi) \in \Gamma\}$. Therefore we shall deal mostly with B.t's or sets of them rather than inequalities, and identify a set $X$ of B.t's with $\{\wedge \varnothing \leqslant \chi \mid \chi \in X\}$, and a single B.t. with its unit set. Thus we write $X \vdash \varphi \leqslant \psi, \varphi \equiv \psi(\sigma)$ etc., and say 
that $\sigma$ is confining when $\{\wedge \varnothing \leqslant \sigma\}$ is confining.

5. State descriptions and independent sequences. For two-valued models as functions into 2 see Definition 16 in the Appendix. Note that if $x$ : $\operatorname{dom}(x)$ $\rightarrow 2, x \vDash \varphi$ then $\varphi$ is supported by $\operatorname{dom}(x)$.

In this section $D$ is a fixed set, and $D_{2}=\{x \mid x: D \rightarrow 2\}$. For each B.t. $\varphi$ let $\operatorname{Mod}_{D}(\varphi)$ be the set of restrictions to $D$ of models of $\varphi$, that is

$$
\operatorname{Mod}_{D}(\varphi)=\left\{x \in D^{D} 2 \mid \exists y[y: D \cup \operatorname{Supp}(\varphi) \rightarrow 2 \text { and } y \mid=\varphi \text { and } x=y \mid D]\right\} \text {. }
$$

Since $D$ is fixed we simply write $\operatorname{Mod}(\varphi)$. If $\varphi$ is supported by $D$ then clearly

$$
\operatorname{Mod}(\varphi)=\left\{x \in \in^{D} 2|x|=\varphi\right\} .
$$

For each $x \in{ }^{D} 2$ let $s d(x)$ be the B.t. $\bigwedge_{i \in D} \pi_{i}$ where $\pi_{i}=p_{i}$ if $x(i)=1, \pi_{i}=7 p_{i}$ if $x(i)=0$. We call $s d(x)$ the state-description of $x$ (the term is due to Carnap), because it specifies completely which atomic B.t's are true and which are false in $x$. It is easy to verify that if $x \in D_{2}$ and $\varphi$ is supported by $D$ then $x \vDash \varphi \Longleftrightarrow \vdash s d(x) \leqslant \varphi, x \not \varphi \Longleftrightarrow \vdash s d(x) \leqslant \neg \varphi$. When $\varphi$ is not necessarily supported by $D$, it will still be true in all cases of interest (but not always) that $\operatorname{Mod}(\varphi)=\operatorname{Mod}^{\prime}(\varphi)$ where $\operatorname{Mod}^{\prime}(\varphi)=\left\{x \in \in^{D} \mid \forall \varphi \wedge s d(x)\right.$ $\leqslant \bigvee \varnothing\}$.

Let us say that $\sigma$ is strongly confining on $D$ when there is a set $E \subseteq D_{2}$ such that $\vdash_{\sigma} \leqslant \bigvee_{x \in E} s d(x)$. If such an $E$ exists, then there is a smallest one, namely $\operatorname{Mod}^{\prime}(\sigma)$. Thus $\sigma$ is strongly confining on $D$ iff $t_{\sigma} \leqslant \bigvee_{x \in \operatorname{Mod}^{\prime}(\sigma)} s d(x)$. When $\sigma$ is supported by $D, \operatorname{tsd}(x) \leqslant \sigma$ for all $x \in \operatorname{Mod}(\sigma)$, so $\sigma$ is strongly confining on $D$ iff $\sigma \equiv \bigvee_{x \in \operatorname{Mod}(\sigma)} \operatorname{sd}(x)$.

5.1. Lemma. If the B.t. $\sigma$ is strongly confining on $D$ then it is confining on $D$ and $B P_{D}(\sigma)$ (the algebra of $\sigma-B o o l e a n$ polynomials over $D$ ) is isomorphic to $P\left(\operatorname{Mod}^{\prime}(\sigma)\right.$ ) (the B.a. of all subsets of $\operatorname{Mod}^{\prime}(\sigma)$ ).

Proof. For any $A \subseteq \operatorname{Mod}^{\prime}(\sigma)$ consider the B.t. $\chi(A)=\bigvee_{x \in A} s d(x)$. Clearly $A \subseteq B \subseteq \operatorname{Mod}^{\prime}(\sigma) \Rightarrow \vdash \chi(A) \leqslant \chi(B)$. On the other hand if $A, B \subseteq$ $\operatorname{Mod}^{\prime}(\sigma)$ and $x \in A \sim B$ then $\operatorname{tsd}(x) \leqslant \chi(A) \wedge \neg \chi(B)$, hence $\{\chi(A) \leqslant$ $\chi(B)\} \vdash s d(x) \leqslant \bigvee \varnothing$ hence $\sigma H \chi(A) \leqslant \chi(B)$ (otherwise $\sigma \vdash_{s} d(x) \leqslant \bigvee \varnothing$, so $\left.x \notin \operatorname{Mod}^{\prime}(\sigma)\right)$. Thus for all $A, B \subseteq \operatorname{Mod}^{\prime}(\sigma), A \subseteq B \Longleftrightarrow \sigma \vdash \chi(A) \leqslant \chi(B)$ and $A=B \Longleftrightarrow \chi(A) \equiv \chi(B)(\sigma)$.

Now let $\varphi$ be any B.t. supported by $D$. Put $\varphi_{1}=\chi\left(\operatorname{Mod}^{\prime}(\sigma) \cap \operatorname{Mod}(\varphi)\right)$, $\varphi_{2}=\chi\left(\operatorname{Mod}^{\prime}(\sigma) \sim \operatorname{Mod}(\varphi)\right)$. Since $\sigma$ is strongly confining, $t_{\sigma} \leqslant \varphi_{1} \vee \varphi_{2}$. But clearly $\vdash \varphi_{1} \leqslant \varphi, \vdash \varphi_{2} \leqslant 7 \varphi$ hence $\varphi \equiv \varphi_{1}(\sigma)$. Thus, for all $\varphi, \psi$ supported 
by $D,\left[\varphi \equiv \chi\left(\operatorname{Mod}^{\prime}(\sigma) \cap \operatorname{Mod}(\varphi)\right)\right](\sigma),\left[\psi \equiv \chi\left(\operatorname{Mod}^{\prime}(\sigma) \cap \operatorname{Mod}(\psi)\right)\right](\sigma)$, and hence by the above $\varphi \equiv \psi(\sigma)$ iff $\operatorname{Mod}^{\prime}(\sigma) \cap \operatorname{Mod}(\varphi)=\operatorname{Mod}^{\prime}(\sigma) \cap \operatorname{Mod}(\psi)$.

Consider now the valuation $(B, D)$ where $B$ is the B.a. of all subsets of $\operatorname{Mod}^{\prime}(\sigma)$ and $I=\left\langle\operatorname{Mod}^{\prime}(\sigma) \cap \operatorname{Mod}\left(p_{i}\right) \mid i \in D\right\rangle$. It is clear by induction on $\varphi$ that if $\varphi$ is supported by $D$ then $\|\varphi\|_{B, I}=\operatorname{Mod}^{\prime}(\sigma) \cap \operatorname{Mod}(\varphi)$. In particular $A \subseteq \operatorname{Mod}^{\prime}(\sigma) \Rightarrow A=\|\chi(A)\|_{B, I}$ and (by the above) $\varphi \equiv \psi(\sigma) \Longleftrightarrow\|\varphi\|_{B, I}=\|\psi\|_{B, I^{*}}$ Thus $(B, I)$ satisfies 4.1(3) (for $\sigma$ in place of $\Gamma$ ), and by $4.1 \sigma$ is confining on $D$ and $B \cong B P_{D}(\sigma)$. This completes the proof.

Now that we have a sufficient condition for being confining on $D$, we shall give a sufficient condition for the opposite case. We use the notation $\psi(\cdot)$ for substitution as defined in the Appendix, Definition 4.

5.2. Definition. Let $\bar{\varphi}=\left\langle\varphi_{N} \mid N \in \Omega\right\rangle$ be any family of B.t's $(\Omega$ is arbitrary) and let $\sigma$ be a B.t. We say that $\bar{\varphi}$ is independent below $\sigma$ when for every B.t. $\psi$ supported by $\Omega, \forall \psi \leqslant \bigvee \varnothing \Rightarrow H_{\sigma} \wedge(\psi(\bar{\varphi})) \leqslant \bigvee_{\varnothing}$

An equivalent definition is: $\bar{\varphi}$ is independent below $\sigma$ when, for every two B.t's $\psi_{1}, \psi_{2}$ supported by $\Omega,\left(\psi_{1}(\bar{\varphi}) \equiv \psi_{2}(\bar{\varphi})\right)(\sigma) \Rightarrow \psi_{1} \equiv \psi_{2}$.

5.3. LEMMA. If there is an infinite family of B.t's supported by $D$ which is independent below $\sigma$, then $\sigma$ is not confining on $D$.

Proof. Let $\bar{\varphi}=\left\langle\varphi_{N} \mid N \in \Omega\right\rangle$ be independent below $\sigma$, and suppose that $\Omega$ is infinite and $\operatorname{Supp}\left(\varphi_{N}\right) \subseteq D$ for each $N$. For any cardinal $\nu$ we can find, by the Gaifman-Hales theorem, a family $\left\langle\psi_{\alpha}\right| \alpha\langle\nu\rangle$ of B.t's supported by $\Omega$ such that no two of them are equivalent. Thus $\alpha, \beta<\nu, \alpha \neq \beta \Rightarrow$ $\forall \psi_{\alpha} \Delta \psi_{\beta} \leqslant \bigvee \varnothing$ where $\psi \Delta \psi^{\prime}=\left(\psi \wedge \neg \psi^{\prime}\right) \vee\left(\psi^{\prime} \wedge \neg \psi\right)$. By the independence of $\bar{\varphi}$ below $\sigma$ we get:

$$
\alpha \neq \beta \Rightarrow H_{\sigma} \wedge\left(\psi_{\alpha} \Delta \psi_{\beta}\right)(\bar{\varphi}) \leqslant \bigvee \varnothing,
$$

i.e., $\alpha \neq \beta \Rightarrow\left(\psi_{\alpha}(\bar{\varphi}) \not \equiv \psi_{\beta}(\bar{\varphi})\right)(\sigma)$. Thus $\left\langle\psi_{\alpha}(\bar{\varphi})\right| \alpha\langle\nu\rangle$ is a family of $\nu$ B.t's supported by $D$ no two of which are equivalent modulo $\sigma$. Since $\nu$ is an arbitrary cardinal, $\sigma$ is nonconfining on $D$.

In the next section we shall apply 5.1 and 5.3 to get a necessary and sufficient condition for $\sigma$ to be confining, assuming $|\operatorname{Sub}(\sigma)| \leqslant \aleph_{0}$.

By the remarks at the end of $\S 4$ this will solve the problem also for any set $\Gamma$ of inequalities such that $|\operatorname{Sub}(\Gamma)| \leqslant \aleph_{0}$, because then $\sigma=\wedge\{\varphi \rightarrow$ $\psi \mid(\varphi \leqslant \psi) \in \Gamma\}$ is equivalent to $\Gamma$ and $|\operatorname{Sub}(\sigma)| \leqslant \aleph_{0}$.

6. A necessary and sufficient condition. The aim of this section is to prove the following.

6.1. Theorem. Let $D$ be a set, $\sigma$ a countable B.t. 
(a) If $\left|\operatorname{Mod}_{D}(\sigma)\right| \leqslant \aleph_{0}$ then $\sigma$ is strongly confining hence confining on $D$, and $B P_{D}(\sigma)$ is isomorphic to the B.a. of all subsets of $\operatorname{Mod}_{D}(\sigma)$.

(b) If $\left|\operatorname{Mod}_{D}(\sigma)\right|>\aleph_{0}$ then $\left|\operatorname{Mod}_{D}(\sigma)\right| \geqslant 2 \aleph_{0}$ and there is an w-sequence of B.t's supported by $D$ which is independent below $\sigma$, hence $\sigma$ is not confining on $D$.

(c) $\sigma$ is confining on $D$ iff $\left|\operatorname{Mod}_{D}(\sigma)\right| \leqslant \aleph_{0}$. Particular cases are:

(d) If $D$ is finite then $\left|\operatorname{Mod}_{D}(\sigma)\right|<\aleph_{0}$ and the assertion of (a) holds.

(e) If $D \sim \operatorname{Supp}(\sigma)$ is infinite (in particular-if $D$ is uncountable) then either $\sigma$ has no models, in which case the assertion of (a) holds with $\operatorname{Mod}_{D}(\sigma)$ $=\varnothing$, or $\operatorname{Mod}_{D}(\sigma)>\aleph_{0}$ and the assertion of (b) holds.

In the proof we shall use the countable completeness theorem for the propositional language, which is proved for our calculus of inequalities in the same way as for the usual systems, e.g. by the Rasiowa-Sikorski lemma.

It can be stated as follows:

If $|\operatorname{Sub}(\varphi \leqslant \psi)| \leqslant \aleph_{0}$ and the inequality $\varphi \leqslant \psi$ holds in every valuation $(\overline{2}, x)$ in which it is defined, then $\vdash_{\varphi} \leqslant \psi$. An alternative formulation is this: Let $D$ be any set, and $\varphi, \psi$ countable B.t's supported by $D$. Then $r_{\varphi} \leqslant \psi$ iff $\operatorname{Mod}(\varphi) \subseteq \operatorname{Mod}(\psi)$ (hence $\varphi \equiv \psi$ iff $\operatorname{Mod}(\varphi)=\operatorname{Mod}(\psi)$ ). Here and below, Mod is always $\operatorname{Mod}_{D}$.

The theorem implies that $|\operatorname{Sub}(\sigma)| \leqslant \aleph_{0} \Rightarrow \operatorname{Mod}(\sigma)=\operatorname{Mod}^{\prime}(\sigma)$ where Mod, Mod' (w.r. to the set $D$ ) are defined in $\S 5$. Indeed, suppose $\sigma$ countable, and then $S=D \cap \operatorname{Supp}(\sigma)$ is a countable set. Putting $1 \cdot p_{i}=p_{i}, 0 \cdot p_{i}=$ $\neg p_{i}$ we have for each $x \in D_{2}$

$$
s d(x)=\bigwedge_{i \in D} x(i) \cdot p_{i}
$$

and

$$
\begin{aligned}
x \in \operatorname{Mod}(\sigma) & \Longleftrightarrow \sigma \wedge s d(x) \text { has a model } \\
& \Longleftrightarrow \sigma \wedge \bigwedge_{i \in S} x(i) \cdot p_{i} \text { has a model; } \\
x \in \operatorname{Mod}^{\prime}(\sigma) & \Longleftrightarrow \forall \sigma \wedge s d(x) \leqslant \vee \varnothing \\
& \Longleftrightarrow H \sigma \wedge \wedge \bigwedge_{i \in S} x(i) \cdot p_{i} \leqslant \vee \varnothing
\end{aligned}
$$

Thus by the countability $x \in \operatorname{Mod}(\sigma) \Longleftrightarrow x \in \operatorname{Mod}^{\prime}(\sigma)$. So $\operatorname{Mod}(\sigma)=\operatorname{Mod}^{\prime}(\sigma)$.

Note that $\{\operatorname{Mod}(\varphi) \mid \varphi$ supported by $D$ and countably long is just the $\sigma$-field of subsets of $D_{2}$ generated by the sets $\operatorname{Mod}\left(p_{i}\right)=\left\{x \in{ }^{D} 2 \mid x(i)=1\right\}$, $i \in D$. The members of this $\sigma$-field are called the Borel subsets of $D_{2}$. The fact 
that for countable $\varphi, \psi$ supported by $D, \operatorname{Mod}(\varphi)=\operatorname{Mod}(\psi)$ iff $\varphi \equiv \psi$ can also be expressed thus: The Borel $\sigma$-field on $D_{2}$ is isomorphic (viewed as a B.a.) to $F^{<r_{1}}(D)$-the free $\sigma$-algebra over $D$. The isomorphism is given by $\operatorname{Mod}(\varphi) \mapsto[\varphi]$ where $[\varphi]$ is the 三-equivalence class of $\varphi\left(F^{<\kappa_{1}}(D)\right.$ is defined by dividing the countable B.t's over $D$ by the equivalence relation $\equiv$ ). All these forms of the countable completeness theorem are well known.

We shall also need a result about analytic subsets of $\omega_{2}$. These are the sets of the form $\{y|\omega| y \in A\}$ where $A$ is a Borel subset of $\omega+\omega_{2}$ (which can be identified with $\omega_{2} \times \omega_{2}$ ). Thus, an analytic set is the projection of a two-dimensional Borel set. The Cantor space $\omega_{2}$ is naturally a topological space-the product of $\aleph_{0}$ discrete two-point spaces. We shall use the fact that every uncountable analytic set in the space $\omega_{2}$ has a closed subset homeomorphic to $\omega_{2}$. For this, as well as several other results about Borel sets in $\boldsymbol{\omega}_{2}$ needed in the next section, the reader can consult volume I of Kuratowski's topology book, but a shorter account which includes just the material we need can be found in [Pa, Chapter I, §§1-3, pp. 1-22].

We are now ready for the proof of 6.1. Note that (c) follows from (a), (b).

Case I. $D$ is finite. Then $\operatorname{Mod}_{D}(\sigma) \subseteq D_{2}$ is finite, and $\vdash \wedge \varnothing \leqslant$ $\bigvee_{x \in D_{2}} s d(x)$ (because this is a finite inequality that holds in all two-valued models); hence $\vdash_{\sigma} \leqslant \bigvee_{x \in D_{2}} s d(x)$ so $\sigma$ is strongly confining on $D$. The rest of (a) follows from 5.1. Thus (a), (d) are proved while (b) and (e) hold vacuously.

Case II. $E=D \sim \operatorname{Supp}(\sigma)$ is infinite. If $\sigma$ has no models, then by the countable completeness theorem $\vdash \sigma \leqslant \bigvee \varnothing$ and so $\sigma$ is strongly confining, $\operatorname{Mod}(\sigma)=\varnothing$. Now suppose that $\sigma$ has at least one model $y_{0}$. We may assume that $y_{0}: \operatorname{Supp}(\sigma) \rightarrow 2$. For any $z: E \rightarrow 2, y_{0} \cup z \mid=\sigma$ and so $\left(\left(y_{0} \mid D\right) \cup\right.$ z) $\in \operatorname{Mod}(\sigma)$. It follows that $|\operatorname{Mod}(\sigma)| \geqslant||^{E} \mid \geqslant 2^{\aleph_{0}}>\aleph_{0}$. Let $e_{n}(n<\omega)$ be distinct elements of $E$ and $\bar{\varphi}=\left\langle p_{e_{n}}\right| n\langle\omega\rangle$. We assert that $\frac{n}{\varphi}$ is independent below $\sigma$. To prove this let $\psi$ be supported by $\omega, H \psi \leqslant \vee \varnothing$. We must show that $H \sigma \wedge \psi(\bar{\varphi}) \leqslant \bigvee \varnothing$. Since $H \psi \leqslant \bigvee \varnothing$ there is a valuation $(B, I)$ such that $I: \omega \rightarrow B$ and $\|\psi\|_{B, I}>0$. It follows that $\|\psi(\bar{\varphi})\|_{B, J_{1}}>0$ where $J_{1}:\left\{e_{n} \mid n<\omega\right\} \rightarrow B$ is defined by $J_{1}\left(e_{n}\right)=I(n)$. But clearly $\|\sigma\|_{B, J_{2}}$ $=1$ where $J_{2}: \operatorname{Supp}(\sigma) \rightarrow B$ is defined by $J_{2}(x)=1^{B}$ if $y_{0}(x)=1, J_{2}(x)$ $=0^{B}$ if $y_{0}(x)=0(x \in \operatorname{Supp}(\sigma))$. Now $J_{1}, J_{2}$ are functions into $B$ with disjoint domains so $\|\sigma \wedge \psi(\bar{\varphi})\|_{B, J_{1} \cup J_{2}}=\|\psi(\bar{\varphi})\|_{B, J_{1}}>0$; hence $\forall \sigma \wedge \psi(\bar{\varphi}) \leqslant$ $\vee \varnothing$. Thus (b) and (e) hold and vacuously also (a) and (d). This completes the proof for Case II.

Case III. $D$ is infinite and countable. This is the most interesting case. Without loss of generality we may assume that $D=\omega$ and $\operatorname{Supp}(\sigma) \subseteq \omega+\omega$. Then $\operatorname{Mod}_{\omega+\omega}(\sigma)$ is a Borel subset of $\omega+\omega_{2}$ and $\operatorname{Mod}(\sigma)=\operatorname{Mod}_{\omega}(\sigma)=$ 
$\left\{y|\omega| y \in \operatorname{Mod}_{\omega+\omega}(\sigma)\right\}$ is an analytic subset of $\omega_{2}$.

(a) Assume that $|\operatorname{Mod}(\sigma)| \leqslant \aleph_{0}$ and consider the inequality $\sigma \leqslant$ $\mathrm{V}_{x \in \operatorname{Mod}(\sigma)^{s d}} d(x)$. This inequality is of countable length and holds in every twovalued model $y \in \omega+\omega_{2} \quad(y|=\sigma \Rightarrow y| \omega \in \operatorname{Mod}(\sigma) \Rightarrow y \mid=s d(x)$ for some $x \in$ $\operatorname{Mod}(\sigma))$. By the countable completeness theorem $\vdash \sigma \leqslant \bigvee_{x \in \operatorname{Mod}(\sigma)^{s d}}(x)$. Therefore $\sigma$ is strongly confining and the rest of (a) follows from 5.1.

(b) $|\operatorname{Mod}(\sigma)|>\aleph_{0}$. Then $\operatorname{Mod}(\sigma)$ is an uncountable analytic set and therefore it has a closed subset $C$ homeomorphic to $\omega_{2}$. Thus $|\operatorname{Mod}(\sigma)| \geqslant$ $|C|=2^{\aleph_{0}}$. Let $F: C \rightarrow \omega_{2}$ be a homeomorphism and for every $n$ let $A_{n}=$

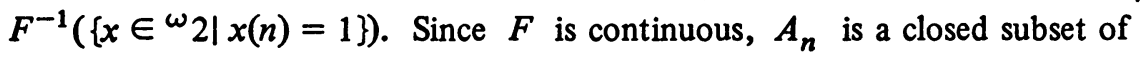
the subspace $C$ of $\omega_{2}$, but $C$ itself is closed so that $A_{n}$ is a closed subset of $\omega_{2}$. In particular, $A_{n}$ is Borel and so there is some countable B.t. $\varphi_{n}$ supported by $\omega$ such that $A_{n}=\operatorname{Mod}\left(\varphi_{n}\right)$. Let us agree to write $1 \cdot \varphi$ for $\varphi$ and $0 \cdot \varphi$ for $7 \varphi$. By definition we have (where Mod is always $\operatorname{Mod}_{\omega}$ ): $F^{-1}\left(\operatorname{Mod}\left(p_{n}\right)\right)=A_{n}=\operatorname{Mod}\left(\varphi_{n}\right)$ and so $F^{-1}\left(\operatorname{Mod}\left(\neg p_{n}\right)\right)=C \sim A_{n}=C \cap$ $\operatorname{Mod}\left(7 \varphi_{n}\right)$. It follows easily that if $y \in \omega_{2}$,

$$
F^{-1}\{y\}=F^{-1}\left(\bigcap_{n} \operatorname{Mod}\left(y(n) \cdot p_{n}\right)\right)=C \cap \operatorname{Mod}\left(\bigwedge_{n} y(n) \cdot \varphi_{n}\right) .
$$

But $F$ is onto, hence $F^{-1}\{y\} \neq \varnothing$, so $\operatorname{Mod}(\sigma) \cap \operatorname{Mod}\left(\bigwedge_{n} y(n) \cdot \varphi_{n}\right) \neq \varnothing$ (for any $y \in \omega_{2}$ ). The proof of (b) will therefore be complete when we prove

6.2. Lemma. Let $\sigma$ be a countable B.t. supported by $\omega+\omega$, and $\bar{\varphi}=\left\langle\varphi_{n}\right| n\langle\omega\rangle$ a sequence of countable B.t's supported by $\omega$. If, for all $y \in \omega_{2}, \operatorname{Mod}\left(\sigma \wedge \wedge_{n} y(n) \cdot \varphi_{n}\right) \neq \varnothing$, then $\bar{\varphi}$ is independent below $\sigma$.

Proof. We must show that if $\psi$ is a B.t. supported by $\omega$ and $H \psi \leqslant$ $\bigvee \varnothing$, then $H \sigma \wedge \psi(\bar{\varphi}) \leqslant \bigvee \varnothing$. First let us prove this when $\psi$ is countable. Since $H \psi \leqslant \bigvee \varnothing, \operatorname{Mod}(\psi) \neq \varnothing$ (by the countable completeness theorem; Mod = $\left.\operatorname{Mod}_{\omega}\right)$. Choose $y \in \operatorname{Mod}(\psi)$. Then $t_{s} d(y) \leqslant \psi$; hence, substituting $\varphi_{n}$ for $p_{n}($ all $n)$ in this inequality, we get (since $\left.s d(y)=\bigwedge_{n} y(n) \cdot p_{n}\right) \vdash \bigwedge_{n} y(n) \cdot$ $\varphi_{n} \leqslant \psi(\bar{\varphi})$. Therefore, $\vdash_{\sigma} \wedge \wedge_{n} y(n) \cdot \varphi_{n} \leqslant \sigma \wedge \psi(\bar{\varphi})$. But $\sigma \wedge \wedge_{n} y(n) \cdot \varphi_{n}$ has models; hence $\sigma \wedge \psi(\bar{\varphi})$ has, hence $H_{\sigma} \wedge \psi(\bar{\varphi}) \leqslant \bigvee \varnothing$.

To prove the assertion for general $\psi$ assume for contradiction that it is false, that is $\exists x\left[x\right.$ is a B.t. supported by $\omega$ and $H x \leqslant \bigvee \varnothing$ and $t_{\sigma} \wedge x(\bar{\varphi})$ $\leqslant \bigvee \varnothing]$. The statement in square brackets is $\Sigma_{1}^{Z F}$ in the variables $\sigma, \bar{\varphi}, x$, and by the assumptions in the lemma $\sigma, \bar{\varphi} \in H C$. By Lévy's theorem it follows that an $x \in H C$ can be found which satisfies the statement in square brackets, but this $x$ would have to be a countable B.t. (call it $\psi$ ), and we have already refuted the existence of such a $\psi$. This contradiction completes the proof of the lemma. 
We have thus proved part (b) of 6.1. (d) and (e) are covered by Cases I, II, and so the proof of 6.1 is complete.

7. Some properties of analytic mappings. The following sections are devoted to a study of the B.a's $F / \sigma$ obtained by dividing all B.t's supported by $\omega$ of length $<k$ (where $k$ is any fixed uncountable regular cardinal) by the equivalence relation $\equiv(\sigma)$ induced by a countable B.t. $\sigma$ (not necessarily supported by $\omega)$. This includes the free $<k$-complete B.a. on $\aleph_{0}$ generators $F$ as a particular case $(\sigma=\bigwedge \varnothing)$. It turns out that the aspects with which we shall deal (e.g. whether $F / \sigma$ is small or large, isomorphic to $F$ or not, whether automorphisms of $F$ with some properties exist or not) require for their study in our approach both Lévy's theorem and some nontrivial known results of descriptive set theory. The connection with descriptive set theory is due to the fact that when $k=\aleph_{1}, F$ is isomorphic to the Borel $\sigma$-field on $\omega_{2}$ (as a B.a.), and then most of the questions are answered by some classical theorems about analytic sets and measurable functions, which will be presented in this section. In extending the results to larger $k$, Lévy's theorem is the main tool.

We consider subsets of $\omega_{2}$. Let Bor be the $\sigma$-field of Borel sets, and for each $X \subseteq \omega_{2}$ put $\operatorname{Bor} / X=\{X \cap B \mid B \in$ Bor $\}$ (the relative Borel $\sigma$-field on $X$, regarded also as a $\sigma$-B.a.). A mapping is for us a triple $(X, F, Y)$ where $F$ is a function from $X$ into $Y$ (we often denote the mapping by $F$ or write $F: X \rightarrow Y$ ). A mapping $F: X \rightarrow Y$ is measurable when for all $z, Z \in \operatorname{Bor} / Y$ $\Rightarrow F^{-1}(Z) \in \operatorname{Bor} / X$, equivalently when for every Borel set $B, F^{-1}(B)$ is Borel relative to $\operatorname{dom}(F)(=X)$. We shall also say then that $F$ (in itself) is a measurable function.

Denote $P_{n}=\left\{x \in \omega^{\omega} \mid x(n)=1\right\}$. For each sequence $\bar{A}=\left\langle A_{n}\right| n\langle\omega\rangle$ of sets there is a unique mapping $F: \omega_{2} \rightarrow \omega_{2}$ satisfying $F^{-1}\left(P_{n}\right)=A_{n}$ for each $n$. We denote this $F$ by $R_{\bar{A}}$ and for each $X \subseteq \omega_{2}$ let $R_{X, \bar{A}}=R_{\bar{A}} \mid X$. If each $A_{n}$ is Borel then $R_{X, \bar{A}}$ is a measurable function.

Every measurable mapping $F: X \rightarrow Y$. induces a $\sigma$-homomorphism $\hat{F}$ : Bor $/ Y \rightarrow$ Bor $/ X$ (the prefix " $\sigma$ " means " $<\aleph_{1}$-complete"), namely $\hat{F}=$ $\left\langle F^{-1}(Z)\right| Z \in$ Bor $\left./ Y\right\rangle$. We shall now show that all $\sigma$-homomorphisms Bor $/ Y \rightarrow$ Bor $/ X$ are obtained in this way.

Let $h: \operatorname{Bor} / Y \rightarrow \operatorname{Bor} / X$ be a $\sigma$-homomorphism. Choose Borel sets $A_{n}$ so that $h\left(Y \cap P_{n}\right)=X \cap A_{n}$ for each $n$ and let $\bar{A}=\left\langle A_{n}\right| n\langle\omega\rangle$. By induction on Borel sets it is easy to see that $h(Y \cap B)=X \cap\left(R_{\bar{A}}\right)^{-1}(B)$ for every Borel set $B$. Thus for every $y \in \omega_{2}$, if $y \notin Y$ then $X \cap\left(R_{\bar{A}}\right)^{-1}\{y\}=h(\not)=$ $\varnothing$; hence $y \notin R_{\bar{A}}^{\prime \prime} X$ (the image of $X$ under $R_{\bar{A}}$ ). Thus $R_{\bar{A}}^{\prime \prime} X \subseteq Y$. Letting $F=R_{X, \bar{A}}$ we get $F: X \rightarrow Y$ and $h(Y \cap B)=F^{-1}(B)=F^{-1}(Y \cap B)$ for 
every Borel set $B$. Thus $F$ is measurable and $h=\hat{F}$.

Note that a measurable mapping $F: X \rightarrow Y$ is onto $(\operatorname{range}(F)=Y$ ) iff $\hat{F}$ is one-to-one. Also, $1_{X}: X \rightarrow X$ is measurable, and if $F: X \rightarrow Y$ and $G: Y \rightarrow Z$ are measurable then $G \circ F: X \rightarrow Z$ is measurable. We have

$$
\hat{1}_{X}=1_{\text {Bor } / X},(G \circ F) \hat{F} \circ \hat{G}, \quad F \neq G \Rightarrow \hat{F} \neq \hat{G} .
$$

These remarks show that the category $M$ of subsets of $\omega_{2}$ with measurable mappings as morphisms is anti-isomorphic to the category of B.a's Bor/X $\left(X \subseteq{ }^{\omega} 2\right)$ with $\sigma$-homomorphisms by the contravariant functor ${ }^{\wedge}$ given by: $X \longmapsto \operatorname{Bor} / X, F \mapsto \hat{F}$. Therefore we shall denote the latter category by $\hat{\mathbf{M}}$. By a measurable isomorphism between $X$ and $Y\left(X, Y \subseteq{ }^{\omega} 2\right)$ we mean an isomorphism (i.e., invertible morphism) in $M$. If $F$ is a measurable isomorphism then $\hat{F}$ is an isomorphism of B.a's.

We shall mainly be interested in the full subcategory $\mathbf{A}$ of $\mathbf{M}$ whose objects are the analytic sets and whose morphisms are all analytic mappings, i.e., measurable mappings $F: X \rightarrow Y$ where $X$ and $Y$ are analytic. The measurable functions $F$ such that $\operatorname{dom}(F)$ is analytic will be called analytic functions. The corresponding subcategory $\hat{\mathrm{A}}$ of $\hat{\mathrm{M}}$ consists of the B.a's Bor/A ( $A$ analytic) and their $\sigma$-homomorphisms.

If in the preceding paragraph we replace the word "analytic" by "Borel" we get the definitions of Borel mappings and functions and of the full subcategories $\mathbf{B}$ and $\hat{\mathbf{B}}$ of $\mathbf{A}$ and $\hat{\mathbf{A}}$.

For information about Borel and analytic sets which we shall use here see [Pa, Chapter I, §§1-3]. Some basic facts about functions are these: If $F$ is an analytic function then $F^{\prime \prime} A$ and $F^{-1}(A)$ are analytic sets for every analytic set $A$. Every analytic set is the range of some Borel function (= measurable function whose domain is Borel); hence every nonempty analytic set is the range of some total Borel function (= measurable function with domain $\omega_{2}$ ). To get less trivial results we need the separation theorem: If $A$ and $B$ are disjoint analytic sets then there is a Borel set $C$ that separates them (i.e. $(A \subseteq C, B \subseteq$ $\sim C)$ ). A simple corollary is that if $F$ is a function such that $F^{-1}(A)$ is analytic for every analytic set $A$ then $F$ is analytic. A more important application is this.

7.1. Lemma. A one-to-one and onto analytic mapping is an isomorphism in A (hence a one-to-one and onto Borel mapping is an isomorphism in $\mathbf{B}$ ).

Proof. Let $F: X \rightarrow Y$ be a one-to-one and onto analytic mapping. We have to show that $F^{-1}: Y \rightarrow X$ is measurable (hence analytic); equivalently, for every $Z \in \operatorname{Bor} / X, F^{\prime \prime} Z \in \operatorname{Bor} / Y$. Let $Z=X \cap B, B \in$ Bor. Since $F$ is 
one-to-one $F^{\prime \prime} Z$ and $F^{\prime \prime}(X \cap \sim B)$ are two disjoint analytic sets whose union is $Y$; hence by separation $F^{\prime \prime} Z$ is Borel relative to $Y$. Q.E.D.

7.2. Corollary. An analytic mapping $F$ is one-to-one iff ${ }^{\prime} \hat{F}$ is onto.

Proof. Let $F: X \rightarrow Y$ be a one-to-one analytic mapping, $Z \in \operatorname{Bor} / X$. By the proof of 7.1, $F^{\prime \prime} Z$ is Borel relative to $\operatorname{range}(F)$, i.e., $F^{\prime \prime} Z=\operatorname{range}(F)$ $\cap B$ for some Borel set $B$. Now, $(Y \cap B) \in \operatorname{Bor} / Y$ and $\hat{F}(Y \cap B)=$ $F^{-1}(Y \cap B)=F^{-1}($ range $(F) \cap B)=Z$ (because $F$ is one-to-one) and so $Z \in \operatorname{range}(\hat{F}) . \hat{F}$ is thus onto $\operatorname{Bor} / X$.

Conversely, if $F$ is onto Bor $/ X$ and $p, q \in X, p \neq q$ then $\{p\}=\hat{F}(Z)$ $=F^{-1}(Z)$ for some $Z \in \operatorname{Bor} / Y$ and thus $F(p) \in Z, F(q) \notin Z, F(p) \neq F(q)$. So $F$ is one-to-one.

The isomorphism theorem $[\mathrm{Pa}$, Chapter I, §2] says that between any two uncountable Borel sets there is a measurable isomorphism, so we have

7.3. If $A, B$ are two uncountable Borel sets or two Borel sets of the same power $\nu \leqslant \aleph_{0}$, then $A$ and $B$ are isomorphic in $B$.

What can be said about analytic non-Borel sets? Let $A$ be such a set. $A$ must be uncountable and therefore has a closed subset $P$ homeomorphic to $\boldsymbol{\omega}_{2}$. It follows easily that there exist measurable mappings $F_{1}: \omega_{2} \rightarrow A, F_{2}: A \rightarrow$ $\omega_{2}$ such that $F_{1}$ is one-to-one and $F_{2}$ is onto. By a remark above, there exists also an onto measurable mapping $F_{3}: \omega_{2} \rightarrow A$ and trivially a one-to-one $F_{4}: A \rightarrow \omega_{2}$. But is there a measurable isomorphism between $\omega_{2}$ and $A$ ? The negative answer follows from the Kuratowski theorem asserting that the range of a one-to-one Borel function is a Borel set. We sum up:

7.4. Let $A$ be analytic and non-Borel. Then $\omega_{2}$ and $A$ are not isomorphic in $M$ though each of them has a one-to-one analytic mapping into the other and an analytic mapping onto the other.

I do not know whether every two analytic non-Borel sets are isomorphic.

The Kuratowski theorem can also be used to show that if $F: X \rightarrow Y$ is a one-to-one Borel mapping then $F$ has a left inverse in B (i.e., a Borel mapping $G: Y \rightarrow X$ such that $G \circ F=1_{X}$ ), except when $X=\varnothing \neq Y$.

We now turn to the coding of analytic sets and functions by B.t's, in order to make possible the applications of Lévy's theorem.

8. Coding sets and functions by B.t's. For any set $\Omega$ and infinite cardinal $\kappa$ we put $B T^{<\kappa}(\Omega)=\{\varphi \mid \varphi$ is a B.t. supported by $\Omega$, of length $<\kappa\}$. Also let $B T^{\omega}=B T^{<\aleph_{1}}(\omega)$. We agree that the letters $\varphi, \chi, \psi, \varphi_{0}, \varphi_{1}, \cdots, \psi_{0}, \cdots$ always denote B.t's. supported by $\omega$, while $\pi, \rho, \sigma, \sigma_{0}, \cdots$ vary on arbitrary B.t's. $\bar{\varphi}, \bar{\psi}$ are always the $\omega$-sequence $\left\langle\varphi_{n}\right| n\langle\omega\rangle,\left\langle\psi_{n}\right| n\langle\omega\rangle$. Note that 
$\bar{\varphi} \in H C \Longleftrightarrow$ each $\varphi_{n}$ is countable; $\bar{\varphi} \in H(\kappa) \Rightarrow\left\{\varphi_{n} \mid n<\omega\right\} \subseteq B T^{<\kappa}(\omega)$. "Mod" is always "Mod $\omega$ ". If $\sigma$ is countable then $\operatorname{Mod}(\sigma)$ is an analytic set because by renaming variables we can assume that $\sigma$ is supported by $\omega+\omega$. We shall often assume the weaker condition $\sigma \in H C$.

Let $A \subseteq \omega^{\omega}$. By a code for $A$ as a Borel set we mean any $\varphi \in B T^{\omega}$ such that $A=\operatorname{Mod}(\varphi)$. A code for $A$ as an analytic set is any $\sigma \in H C$ such that $A=\operatorname{Mod}(\sigma)$.

Operations on sets can be represented on their codes as the following equations show (we need not assume countability of the B.t's. to ensure that they hold):

$$
\begin{aligned}
\sim \operatorname{Mod}(\varphi) & =\operatorname{Mod}(\neg \varphi), & & \bigcap_{i} \operatorname{Mod}\left(\varphi_{i}\right)=\operatorname{Mod}\left(\bigwedge_{i} \varphi_{i}\right), \\
\operatorname{Mod}(\sigma) \cap \operatorname{Mod}(\varphi) & =\operatorname{Mod}(\sigma \wedge \varphi), & & \bigcup_{i} \operatorname{Mod}\left(\sigma_{i}\right)=\operatorname{Mod}\left(\bigvee_{i} \sigma_{i}\right) .
\end{aligned}
$$

In general, $\operatorname{Mod}(\neg \sigma) \neq \sim \operatorname{Mod}(\sigma), \operatorname{Mod}(\sigma \wedge \rho) \neq \operatorname{Mod}(\sigma) \cap \operatorname{Mod}(\rho)$.

Intersections of the form $\bigcap_{i \in I} \operatorname{Mod}\left(\sigma_{i}\right)$ can be dealt with as follows: For each $i$ let $\sigma_{i}^{\prime}$ be the result of substituting $p_{(i, x)}$ in $\sigma_{i}$ for each occurrence of a variable $p_{x}, x \notin \omega$. Thus the sets $\operatorname{Supp}\left(\sigma_{i}^{\prime}\right) \sim \omega$ are pairwise disjoint. Denote $M_{i \in I} \sigma_{i}=\bigwedge_{i \in I} \sigma_{i}^{\prime}$. It is easily seen that $\operatorname{Mod}\left(M_{i} \sigma_{i}\right)=\bigcap_{i} \operatorname{Mod}\left(\sigma_{i}\right)$. Note also that if $\sigma_{i}$ is supported by $\omega$ then $\sigma_{i}^{\prime}=\sigma_{i}$.

Coding functions is only slightly more complicated. Given $\bar{\varphi}=\left\langle\varphi_{n}\right| n\langle\omega\rangle$ we let $R_{\bar{\varphi}}$ be the unique function $F: \omega_{2} \rightarrow \omega_{2}$ satisfying $F^{-1}\left(P_{n}\right)=$ $\operatorname{Mod}\left(\varphi_{n}\right)$ for all $n\left(P_{n}=\{x \mid x(n)=1\}\right)$. For any $\sigma$ and $\bar{\varphi}$ put $R_{\sigma, \bar{\varphi}}=$ $R_{\bar{\varphi}} \mid \operatorname{Mod}(\sigma)$ : This is the unique $F: \operatorname{Mod}(\sigma) \rightarrow \omega_{2}$ satisfying $F^{-1}\left(P_{n}\right)=$ $\operatorname{Mod}\left(\sigma \wedge \varphi_{n}\right)$ for all $n$. It is clear that a function $F$ is analytic iff $F=R_{\sigma, \bar{\varphi}}$ for some $(\sigma, \bar{\varphi}) \in H C$ (i.e. $\sigma \in H C, \varphi_{n} \in B T^{\omega}$ for each $n$ ), and $F$ is Borel iff $F=R_{\chi, \bar{\varphi}}$ for some $(\chi, \bar{\varphi}) \in H C \quad(\chi$ supported by $\omega)$. In the notation of $\S 7$, if $X=\operatorname{Mod}(\sigma)$ and $A_{n}=\operatorname{Mod}\left(\varphi_{n}\right)(n<\omega), \bar{A}=\left\langle A_{n}\right| n\langle\omega\rangle$, then $R_{\bar{\varphi}}=R_{\bar{A}}, R_{\sigma, \bar{\varphi}}=R_{X, \bar{A}}$.

For any function $F$, a code for $F$ is a Borel [analytic] function is any pair $(\chi, \bar{\varphi}) \in H C[(\sigma, \bar{\varphi}) \in H C]$ such that $F=R_{\chi, \bar{\varphi}}\left[F=R_{\sigma, \bar{\varphi}}\right.$ resp. $]$.

How can operations on functions be represented on the codes? The most important fact to notice is the equation $\left(R_{\bar{\varphi}}\right)^{-1}(\operatorname{Mod}(\rho))=\operatorname{Mod}(\rho(\bar{\varphi}))$ [recall that $\rho(\bar{\varphi})$ results from $\rho$ by substituting $\varphi_{n}$ for $p_{n}$ (all $n<\omega$ ), without affecting $p_{x}$ for $\left.x \notin \omega\right]$. The proof is not hard, using the semantic meaning of substitution, and is left to the reader. The following lemma collects some facts which are either trivial or follow easily from the above equation. Note that $R_{\sigma, \bar{\varphi}}, R_{\rho, \bar{\psi}}$ are functions, not mappings, so that their composition is always 
defined $(G \circ F=\langle G(F(x)) \mid x \in \operatorname{dom}(F), F(x) \in \operatorname{dom}(G)\rangle)$.

8.1. Lemma. (a) Restriction: $R_{\sigma, \bar{\varphi}} \mid \operatorname{Mod}(\rho)=R_{\sigma \mathbb{M} \rho, \bar{\varphi}}$.

(b) Identity function: $1_{\mathrm{Mod}(\sigma)}=R_{\sigma,\left\langle p_{n} \mid n<\omega\right\rangle}$.

(c) Inverse image: $\left(R_{\bar{\varphi}}\right)^{-1}(\operatorname{Mod}(\rho))=\operatorname{Mod}(\rho(\bar{\varphi}))$ and $\left(R_{\sigma, \bar{\varphi}}\right)^{-1}(\operatorname{Mod}(\rho))=$ $\operatorname{Mod}(\sigma M(\rho(\bar{\varphi})))$.

(d) Composition: Let $\bar{\psi} * \bar{\varphi}=\left\langle\psi_{n}(\bar{\varphi})\right| n\langle\omega\rangle$. Then $R_{\bar{\psi}} \circ R_{\bar{\varphi}}=$ $R_{\bar{\Psi} * \bar{\varphi}}$ and $R_{\rho, \bar{\psi}} \circ R_{\sigma, \bar{\varphi}}=R_{\alpha \mathbb{X} \rho(\bar{\varphi}), \bar{\psi} * \bar{\varphi}}$.

(e) Equality: $R_{\sigma, \bar{\varphi}}=R_{\rho, \bar{\psi}}$ iff $\operatorname{Mod}(\sigma)=\operatorname{Mod}(\rho)$ and for all $n$, $\operatorname{Mod}\left(\sigma \wedge \varphi_{n}\right)=\operatorname{Mod}\left(\rho \wedge \psi_{n}\right)$.

It is also not hard to express $R_{\sigma, \bar{\varphi}}^{\prime \prime} \operatorname{Mod}(\rho)$ as $\operatorname{Mod}(\pi)$ for a suitable $\pi$. We are not assuming countability of the B.t's in 8.1. A natural question is which operations and relations on Borel and analytic objects are "computable" or "decidable" on the codes but we cannot discuss it here (see end of §11).

We proceed to code mappings. A code for $F: A \rightarrow B$ as an analytic mapping is a triple $(\sigma, \bar{\varphi}, \rho)$ such that $(\sigma, \bar{\varphi})$ is a code for $F$ as an analytic function (hence $\sigma$ is a code for $A$ ) and $\rho$ is a code for $B$ as an analytic set. Borel mappings are coded similarly.

Let $(\sigma, \bar{\varphi}, \rho) \in H C$. Is $(\sigma, \bar{\varphi}, \rho)$ a code for some analytic mapping? The condition is that range $\left(R_{\sigma, \bar{\varphi}}\right) \subseteq \operatorname{Mod}(\rho)$, equivalently that $\operatorname{Mod}(\sigma) \subseteq \operatorname{Mod}\left(R_{\bar{\varphi}}\right)^{-1}$ - $(\operatorname{Mod}(\rho))$, i. e., (by 8.1) $\operatorname{Mod}(\sigma) \subseteq \operatorname{Mod}(\rho(\bar{\varphi}))$. By 8.1 the operations in the category $A$ of analytic sets and mapping (passing from $A$ to $1_{A}$; composing two mappings) can be computed on the codes. Similarly for the Borel category $\mathbf{B}$.

Now suppose $(\sigma, \bar{\varphi}, \rho)$ codes $F: X \rightarrow Y$ as an analytic mapping, and let us express $\hat{F}:$ Bor $/ Y \rightarrow \operatorname{Bor} / X$. For every $\chi$ (supported by $\omega$ ) we have $F^{-1}(Y \cap \operatorname{Mod}(\chi))=F^{-1}(\operatorname{Mod}(\chi))=\operatorname{Mod}(\sigma \wedge \chi(\bar{\varphi}))$. Therefore $\hat{F}$ is given by $\hat{F}(\operatorname{Mod}(\rho \wedge \chi))=\operatorname{Mod}(\sigma \wedge \chi(\bar{\varphi}))$ for all $\chi \in B T^{\omega}$. Recall from $\S 7$ that $F$ is onto iff $\hat{F}$ is one-to-one and $F$ is one-to-one iff $\hat{F}$ is onto. This leads to the following equivalences.

8.2. Lemma. Let $\sigma, \bar{\varphi}, \rho \in H C, X=\operatorname{Mod}(\sigma), F=R_{\sigma, \bar{\varphi}}, Y=\operatorname{Mod}(\rho)$, $A_{n}=\operatorname{Mod}\left(\varphi_{n}\right)$ for each $n$. Suppose $F$ is into $Y$ (i.e. $\operatorname{Mod}(\sigma) \subseteq \operatorname{Mod}(\rho(\bar{\varphi}))$. Then the following conditions are equivalent:

(1) $F$ is onto $Y$;

(2) for all $y \in Y, X \cap \bigcap_{n} y_{n} \cdot A_{n} \neq \varnothing$ (equivalently, for all $y \in Y$, $\left.H_{\sigma} \wedge \wedge_{n} y_{n} \cdot \varphi_{n} \leqslant \bigvee \varnothing\right)$ where $1 \cdot A=A, 0 \cdot A=\sim A, 1 \cdot \varphi=\varphi, 0 \cdot \varphi=$ $7 \varphi$;

(3) $\hat{F}$ is one-to-one; 
(4) for all countable $\chi_{1}, \chi_{2}$ (supported by $\left.\omega\right), \chi_{1} \not \equiv \chi_{2}(\rho) \Rightarrow\left[\chi_{1}(\bar{\varphi}) \not \equiv\right.$ $\left.\chi_{2}(\bar{\varphi})\right](\sigma)$

(5) same as (4), omitting the word "countable".

PRoof. (1) $\Longleftrightarrow(2)$ because (2) says that for each $y \in Y, F^{-1}(\{y\}) \neq \varnothing$.

(3) $\Longleftrightarrow$ (4) because (4) says that for all $\chi_{1}, \chi_{2} \in B T^{\omega}, \operatorname{Mod}\left(\rho \wedge \chi_{1}\right) \neq$ $\operatorname{Mod}\left(\rho \wedge \chi_{2}\right) \Rightarrow \hat{F}\left(\operatorname{Mod}\left(\rho \wedge \chi_{1}\right)\right)=\operatorname{Mod}\left(\sigma \wedge \chi_{1}(\bar{\varphi})\right) \neq \operatorname{Mod}\left(\sigma \wedge \chi_{2}(\bar{\varphi})\right)=$ $\hat{F}\left(\operatorname{Mod}\left(\rho \wedge \chi_{2}\right)\right)$.

(1) $\Longleftrightarrow$ (3) we know. (5) $\Rightarrow(4)$ is obvious.

(4) $\Rightarrow(5)$ : If a counterexample to (5) exists, then by Lévy's theorem and the fact that $\sigma, \bar{\varphi}, \rho \in H C$ a counterexample to (4) exists.

8.3. Lemma. Let $\sigma, \bar{\varphi}, \rho, X, F, Y, A_{n}$ be as in 8.2 and let $F$ be into $Y$. The following are equivalent:

(1) $F$ is one-to-one;

(2) for all $y \in \omega_{2},\left|X \cap \bigcap_{n} y_{n} \cdot A_{n}\right| \leqslant 1$;

(3) for all $y \in \omega_{2}, m<\omega: \operatorname{to} \wedge \Lambda_{n} y_{n} \cdot \varphi_{n} \leqslant p_{m}$ or $\vdash_{\sigma} \wedge \Lambda_{n} y_{n}$. $\varphi_{n} \leqslant 7 p_{m} ;$

(4) $\hat{F}$ is onto (Bor/X);

(5) there is a sequence $\bar{\psi}$ of countable B.t's (supported by $\omega$ ) such that $\left(\psi_{n}(\bar{\varphi}) \equiv p_{n}\right)(\sigma)$ for all $n$ (equivalently for each $n$ there is a countable $\psi$ such that $\left.\left(\psi(\bar{\varphi}) \equiv p_{n}\right)(\sigma)\right)$;

(6) same as (5) with the word "countable" omitted.

REMARK. Several other equivalent conditions could be added here, for instance the condition that there is an analytic set $Z(=\operatorname{range}(F))$ and an analytic mapping $G: Z \rightarrow X\left(G=F^{-1}\right)$ such that $G \circ F=1_{X}, F \circ G=1_{Z}$, and various expressions of the same fact by B.t's.

Proof. (1) $\Longleftrightarrow(2) \Longleftrightarrow(3)$. Left to the reader.

(1) $\Longleftrightarrow$ (4). From \$7.

(4) $\Longleftrightarrow$ (5). $\hat{F}$ is onto iff for each $n\left(X \cap P_{n}\right) \in$ range $\hat{F}$ where $P_{n}=$ $\operatorname{Mod}\left(p_{n}\right)$. Thus we need for each $n$ some $\psi \in B T^{\omega}$ such that $\hat{F}(\operatorname{Mod}(\rho \wedge \psi))$ $=\operatorname{Mod}\left(\sigma \wedge p_{n}\right)$, i.e. $\operatorname{Mod}(\sigma \wedge \psi(\bar{\varphi}))=\operatorname{Mod}\left(\sigma \wedge p_{n}\right)$, that is $\left(\psi(\bar{\varphi}) \equiv p_{n}\right)(\sigma)$.

(5) $\Longleftrightarrow(6)$. By Lévy's theorem.

It will be clear to the reader that various other conditions about analytic and Borel mappings can be expressed in a variety of equivalent forms using the codes and Levy's theorem (e.g. being an isomorphism, having a right inverse, etc.).

9. The B.a's $B T^{<\kappa}(\omega) / \sigma \vdash$ for countable $\sigma$. We follow the notations agreed upon in the first paragraph of $\S 8$. Dividing $B T^{<k}(\omega)$ by the equivalence relation $\equiv(\sigma)$ we obtain the "Lindenbaum algebra" $B T^{<\kappa}(\omega) / \sigma \vdash$ (see Appendix, 
Definition 18 and Lemma 3). Our purpose is to study these B.a's for $\kappa>\aleph_{0}$ and countably long $\sigma \quad(\in H C$ without loss of generality).

Under a suitable interpretation many of the results hold for any uncountable $\kappa$ (and, in a theory with proper classes, for $\kappa=\infty$ too). But since this requires special definitions (for instance $<\kappa$-complete homomorphisms have to be replaced for singular $\kappa$ by those which, for any $\lambda<\kappa$, are $<\lambda^{+}$-complete when restricted to the $<\lambda^{+}$-subalgebra of $B T^{<\kappa}(\omega) / \sigma \vdash$ generated by the elements of $B T^{<\kappa}(\omega) / \sigma$ - corresponding to the variables $p_{n}(n<\omega)$ ), and leads to some difficulties when $c f(\kappa)=\omega$, we simplify matters by dealing only with regular $\kappa>\aleph_{0}$ (not much of interest is lost). Our methods and results do not apply to $\kappa=\aleph_{0}$-the B.a's $B T^{<\kappa_{0}}(\omega) / \sigma \vdash$ are countable and their study does not fall within the subject of this work.

From now till the end of $\S 10, k$ is a fixed regular cardinal $>\aleph_{0}$ and $\pi, \rho, \sigma$ are restricted to $H C$. For any $\sigma(\in H C)$ we denote the B.a. $B T^{<\kappa}(\omega) / \sigma \vdash$ by $F / \sigma$ and let $[\chi]_{\sigma}=\left\{\psi \in B T^{<\kappa}(\omega) \mid \chi \equiv \psi(\sigma)\right\}$ for any $\chi \in$ $B T^{<\kappa}(\omega)$. The elements of $F / \sigma$ are the classes $[\chi]_{\sigma}, \chi \in B T^{<\kappa}(\omega)$. When $\sigma=\Lambda \varnothing$ we denote $F / \sigma$ by $F$ and $[\chi]_{\sigma}$ by $[\chi] . F$ is the free $<\kappa$-complete B.a. on $\aleph_{0}$ generators, and $F / \sigma$ can be viewed as the quotient of $F$ by the filter $\left\{[\chi] \mid \chi \in B T^{<\kappa}(\omega), \vdash \sigma \leqslant \chi\right\}$.

By the countable completeness theorem it is clear that for any $\sigma$ the equation $I_{\sigma}(\operatorname{Mod}(\sigma \wedge \varphi))=[\varphi]_{\sigma}\left(\varphi \in B T^{\omega}\right)$ defines a one-to-one function $I_{\sigma}$ : $\operatorname{Bor} / \operatorname{Mod}(\sigma) \rightarrow F / \sigma$, which is in fact a $<\aleph_{1}$-complete embedding. We shall take advantage of this and identify, for each $\sigma \in H C$, the relatively Borel subsets of the analytic set $\operatorname{Mod}(\sigma)$ with the elements of $F / \sigma$ corresponding to them by $I_{\sigma}$. Thus $\operatorname{Bor} / \operatorname{Mod}(\sigma)$ becomes the $\left\langle\aleph_{1}\right.$-subalgebra of $F / \sigma$ generated by $\left\{\left[p_{n}\right]_{\sigma} \mid n<\omega\right\}$, whose underlying set is $\left\{[\varphi]_{\sigma} \mid \varphi \in B T^{\omega}\right\}$. In particular the Borel field itself becomes, under $I_{\bigwedge_{\varnothing}}$, a $<\aleph_{1}$-subalgebra of $F$ (it is the free $\left\langle\aleph_{1}\right.$-complete B.a. on $\aleph_{0}$ generators), and all Borel sets are members of $F$.

The atoms of $F / \sigma$ are just those elements $\left[\bigwedge_{n} x_{n} \cdot p_{n}\right]_{\sigma}\left(x \in \omega_{2}\right)$ which are not zero. Therefore these are just the atoms of $\operatorname{Bor} / \operatorname{Mod}(\sigma)$, and can be identified with points of $\operatorname{Mod}(\sigma)$. It follows that for $A \in \operatorname{Bor} / \operatorname{Mod}(\sigma),|A|$ is just the number of atoms of $F / \sigma$ below $A$.

9.1. Let $\sigma_{1}, \sigma_{2} \in H C, \operatorname{Mod}\left(\sigma_{1}\right)=\operatorname{Mod}\left(\sigma_{2}\right)$. Then for all $\varphi, \psi$ (supported by $\omega) \varphi \equiv \psi\left(\sigma_{1}\right)$ iff $\varphi \equiv \psi\left(\sigma_{2}\right)$. Hence $F / \sigma_{1}=F / \sigma_{2}$.

Proof. We first prove this for $\varphi, \psi \in H C$. By the countable completeness theorem

$$
\begin{aligned}
& \varphi \equiv \psi\left(\sigma_{1}\right) \Longleftrightarrow \operatorname{Mod}\left(\sigma_{1}\right) \cap \operatorname{Mod}(\varphi)=\operatorname{Mod}\left(\sigma_{1}\right) \cap \operatorname{Mod}(\psi) \\
& \Leftrightarrow \operatorname{Mod}\left(\sigma_{2}\right) \cap \operatorname{Mod}(\varphi)=\operatorname{Mod}\left(\sigma_{2}\right) \cap \operatorname{Mod}(\psi) \Longleftrightarrow \varphi \equiv \psi\left(\sigma_{2}\right) .
\end{aligned}
$$


For general $\varphi, \psi$ we get the result from Lévy's theorem:

$$
\exists \varphi \psi\left[\varphi \equiv \psi\left(\sigma_{1}\right) \nLeftarrow \varphi \equiv \psi\left(\sigma_{2}\right)\right] \Rightarrow(\exists \sigma, \psi \in H C)\left[\varphi \equiv \psi\left(\sigma_{1}\right) \Longleftrightarrow \varphi \equiv \psi\left(\sigma_{2}\right)\right] .
$$

The consequent is false; hence so is the antecedent.

In view of 9.1 we define $F / X$ for any analytic set $X$ to be $F / \sigma$ where $\sigma$ is any code for $X$. $F / \omega_{2}$ is $F$ itself. Also, we denote for each $\chi \in B T^{<\kappa}(\omega)$ and analytic set $X,[\chi]_{X}=[\chi]_{\sigma}$ where $\sigma$ is any code for $X$. It follows that from now on we can regard $B o r / X$ as a $\left\langle\aleph_{1}\right.$-subalgebra of $F / X$ for any analytic $X$.

By $\$ 7$ we have got, for any analytic mapping $F: X \rightarrow Y$, a $\sigma$-homomorphism $\hat{F}:$ Bor $/ Y \rightarrow \operatorname{Bor} / X$. We shall now extend $\hat{F}$ to a $<k$-complete homomorphism $F^{*}: F / Y \rightarrow F / X$.

9.2. Let $F: X \rightarrow Y$ be an analytic mapping. Then there is a unique $K$ : $F / Y \rightarrow F / X$, denoted by $F^{*}$, such that $K$ is a $<K$-complete homomorphism and $K$ extends $F$. If $(\sigma, \bar{\varphi}, \rho)$ is any code for $F: X \rightarrow Y$, then $F^{*}$ is given by $F^{*}\left([\chi]_{\rho}\right)=[\chi(\bar{\varphi})]_{\sigma}$ for $\chi \in B T^{<k}(\omega)$.

Proof. Uniqueness of $K$ follows from the fact that Bor $/ Y$ generates $F / Y$ in the $<k$-sense. To prove existence choose any code $(\sigma, \bar{\varphi}, \rho)$ for $F$ and consider the equation $K\left([\chi]_{\rho}\right)=[\chi(\bar{\varphi})]_{\sigma}, \chi \in B T^{<\kappa}(\omega)$.

To prove that it defines a single-valued function we must show that $\chi_{1} \equiv$ $\chi_{2}(\rho) \Rightarrow\left[\chi_{1}(\bar{\varphi}) \equiv \chi_{2}(\bar{\varphi})\right](\sigma)$. But we know from $\S 8$ (just before 8.2) that when $\chi$ is restricted to $B T^{\omega}$ the above equation, which becomes $K(\operatorname{Mod}(\rho \wedge \chi))=$ $\operatorname{Mod}(\sigma \wedge \chi(\bar{\varphi}))$, defines the single-valued function $\hat{F}$. Therefore $\chi_{1} \equiv \chi_{2}(\rho) \Rightarrow$ $\left[\chi_{1}(\bar{\varphi}) \equiv \chi_{2}(\bar{\varphi})\right](\sigma)$ holds for $\chi_{1}, \chi_{2} \in B T^{\omega}$. By Lévy's theorem it must hold for all $\chi_{1}, \chi_{2}$ supported by $\omega$. Thus we have got a function $K: F / \rho \rightarrow F / \sigma$, i.e., $K: F / Y \rightarrow F / X$ which satisfies $K\left([\chi]_{\rho}\right)=[\chi(\bar{\varphi})]_{\sigma}$ for all $\chi \cdot \in B T^{<k}(\omega)$ and extends $\hat{F}$. $K$ is easily seen to be a $<_{\kappa}$-complete homomorphism, and the proof is complete.

By 9.2 we are naturally led to consider the category $A^{*}$ with the $<_{k}$ complete B.a's $F / X$ (all analytic $X$ ) as objects and the < $<$-complete homomorphisms of them as morphisms. Recall also the category $\hat{\mathbf{A}}$ defined in $\S 7$. To each object Bor/X of $\hat{\mathbf{A}}$ corresponds an extension $F / X$ in $\mathbf{A}^{*}$, and 9.2 shows that every morphism $\hat{F}: \operatorname{Bor} / Y \rightarrow \operatorname{Bor} / X$ of $\hat{\mathbf{A}}$ has a unique extension to a morphism $F^{*}: F / Y \rightarrow F / X$ of $A^{*}$. It follows easily from the uniqueness or by a direct computation that $\left(1_{X}\right)^{*}=1_{F / X}$ and that the extension of $\hat{F} \circ \hat{G}$ $(=(G \circ F))$ is $F^{*} \circ G^{*}\left(=(G \circ F)^{*}\right)$. Thus we have a covariant functor $e$ : $\hat{\mathbf{A}} \rightarrow \mathrm{A}^{*}$ given by $e(\operatorname{Bor} / X)=F / X, e(\hat{F})=F^{*}$. Composing $e$ and the antiisomorphism ${ }^{\wedge}$ of $\mathbf{A}$ and $\hat{\mathbf{A}}$ we get a contravariant functor ${ }^{*}: \mathbf{A} \rightarrow \mathbf{A}^{*}$ which takes $X$ to $F / X$ and $F: X \rightarrow Y$ to $F^{*}: F / Y \rightarrow F / X$. 
Morphisms of $\mathrm{A}^{*}$ need not all be of the form $F^{*}$, but we have a similar way, of representing them by sequences of B.t's. For let $f: F / Y \rightarrow F / X$ be a morphism of $\mathrm{A}^{*}$ (so that $X, Y$ are analytic and $f$ is $<k$-complete). Choose $\bar{\varphi} \in H(\kappa)$ so that for each $n f\left(\left[p_{n}\right]_{Y}\right)=\left[\varphi_{n}\right]_{X}$. Then by induction on $\chi$ we get $f\left([\chi]_{Y}\right)=[\chi(\bar{\varphi})]_{X}$ for all $\chi \in B T^{<\kappa}(\omega)$. If $\sigma$ and $\rho$ are codes for $X$ and $Y$ respectively, it follows that for all $\chi_{1}, \chi_{2} \in B T^{<k}(\omega): \chi_{1} \equiv \chi_{2}(\rho) \Rightarrow$ $\left(\chi_{1}(\bar{\varphi}) \equiv \chi_{2}(\bar{\varphi})\right)(\sigma)$. Since $\sigma, \rho, \bar{\varphi} \in H(\kappa)$ (actually $\sigma, \rho \in H C$ ) we get by Lévy's theorem that $\chi_{1} \equiv \chi_{2}(\rho) \Rightarrow\left(\chi_{1}(\bar{\varphi}) \equiv \chi_{2}(\bar{\varphi})\right)(\sigma)$ for all $\chi_{1}, \chi_{2}$ (supported by $\omega)$, not only $\dot{\chi}_{1}, \chi_{2} \in H(\kappa)$.

We shall say, when $\sigma, \rho, \bar{\varphi}$ are chosen as above, that $(\sigma, \bar{\varphi}, \rho)$ is a code for the $\mathrm{A}^{*}$-morphism $f$. Every code $(\sigma, \bar{\varphi}, \rho)$ for an $\mathrm{A}^{*}$-morphism thus satisfies: $\sigma, \rho \in H C, \bar{\varphi} \in H(\kappa)$ and for all $\chi_{1}, \chi_{2}, \chi_{1} \equiv \chi_{2}(\rho) \Rightarrow\left(\chi_{1}(\bar{\varphi}) \equiv \chi_{2}(\bar{\varphi})\right)(\sigma)$. Conversely, if $(\sigma, \bar{\varphi}, \rho)$ satisfies these requirements, then it is a code for a unique $\mathbf{A}^{*}$-morphism $f: F / Y \rightarrow F / X$ given as follows: $X=\operatorname{Mod}(\sigma), Y=\operatorname{Mod}(\rho)$ and $f\left([\chi]_{\rho}\right)=[\chi(\bar{\varphi})]_{\sigma}$ for all $\chi \in B T^{<\kappa}(\omega)$. We shall denote this morphism by $R_{(\sigma, \bar{\varphi}, \rho)}^{*}$. The reader can verify that when $\bar{\varphi} \in H C,(\sigma, \bar{\varphi}, \rho)$ codes an analytic mapping $F: X \rightarrow Y$ just in case it codes an $\mathbf{A}^{*}$-morphism and then this $\mathbf{A}^{*}$ morphism is $F^{*}$. Note also that if $X=\operatorname{Mod}(\sigma)$ then $\left(\sigma,\left\langle p_{n}\right| n\langle\omega\rangle, \sigma\right)$ codes $1_{F / X}$ and that if $(\sigma, \bar{\varphi}, \rho)$ and $(\rho, \bar{\psi}, \pi)$ code $A^{*}$-morphisms then so does $(\sigma, \bar{\psi} * \bar{\varphi}, \pi)$ and we have $R_{(\sigma, \bar{\psi} * \bar{\varphi}, \pi)}^{*}=R_{(\sigma, \bar{\varphi}, \rho)}^{*}{ }^{\circ} R_{(\rho, \bar{\psi}, \pi)}^{*}$ (prove first that $(\chi(\bar{\psi}))(\bar{\varphi})=\chi(\bar{\psi} * \bar{\varphi})$ always). We can also "decide" when two codes represent the same morphism. Clearly $R_{\left(\sigma_{1}, \bar{\varphi}, \rho_{1}\right)}^{*}=R_{\left(\sigma_{2}, \bar{\psi}, \rho_{2}\right)}^{*}$ iff $\operatorname{Mod}\left(\sigma_{1}\right)=$ $\operatorname{Mod}\left(\sigma_{2}\right), \operatorname{Mod}\left(\rho_{1}\right)=\operatorname{Mod}\left(\rho_{2}\right)$ and $\left[\varphi_{n}\right]_{\sigma_{1}}=\left[\psi_{n}\right]_{\sigma_{2}}$ (i.e. $\left.\varphi_{n} \equiv \psi_{n}\left(\sigma_{1}\right)\right)$ for all $n$.

Also note that, by definition, $R_{(\sigma, \bar{\varphi}, \rho)}^{*}$ is one-to-one iff for all $\chi_{1}, \chi_{2} \in$ $B T^{<\kappa}(\omega), \chi_{1} \not \neq \chi_{2}(\rho) \Rightarrow\left(\chi_{1}(\bar{\varphi}) \not \equiv \chi_{2}(\bar{\varphi})\right)(\sigma)$ iff this implication holds for all $\chi_{1}$, $\chi_{2}$ (supported by $\omega$ ). The second "iff" is by Lévy's theorem and the fact that $\sigma, \bar{\varphi}, \rho \in H(\kappa)$.

Also, $R_{(\sigma, \bar{\varphi}, \rho)}^{*}$ is onto iff, for each $n,\left[p_{n}\right]_{\sigma}$ is in its range, iff for each $n$ there exists some $\psi \in B T^{<\kappa}(\omega)$ such that $\left(\psi(\bar{\varphi}) \equiv p_{n}\right)(\sigma)$ iff for each $n$ there exists some $\psi$ (supported by $\omega$ ) such that $\left(\psi(\bar{\varphi}) \equiv p_{n}\right)(\sigma)$. The last "iff" is again by Lévy's theorem.

For the special case when $\bar{\varphi} \in H C$ so that $R_{(\sigma, \bar{\varphi}, \rho)}^{*}$ has the form $F^{*}$ for some analytic mapping $F$ we get for $F^{*}$ being one-to-one or onto exactly the conditions $8.2(5), 8.3(6)$ respectively. This leads to the following supplements tc 8.2 and 8.3 .

9.3. Let $\sigma, \bar{\varphi}, \rho, X, F, Y, A_{n}(n<\omega)$ be as in 8.2 , and let $F$ be into $Y$. Denote $\mathrm{C}=\left[\left\{X \cap A_{n} \mid n<\omega\right\}\right]_{\mathrm{Bor} / X}^{<1_{1}}$ (i.e. the smallest $\sigma$-subalgebra of Bor $/ X$ containing the sets $\left.X \cap A_{n}\right), D=\left[\left\{X \cap A_{n} \mid n<\omega\right\}\right]_{F / K}^{<K}$ (i.e. the smallest 
$<_{\kappa}$-subalgebra of $F / X$ containing $X \cap A_{n}$, which is identified with $\left[\varphi_{n}\right]_{X}$, for each $n$ ).

The following conditions are equivalent to $F$ being onto $Y$ : (1) $-(5)$ as in 8.2;

(6) $F^{*}$ is one-to-one.

When $\rho=\Lambda \varnothing$ (so that $F / Y=F / \omega_{2}=F$ ) one can add:

(7) $C$ is a free $\sigma$-B.a. on the generators $A_{n} \cap X, n<\omega$;

(8) $D$ is a free $<k$-complete B.a. on the same generators.

Proof. The equivalence of (5) and (6) has been noted above. Hence (1)(6) are equivalent. For (7) and (8) note that since for each $n A_{n} \cap X=\hat{F}\left(\left[\rho_{n}\right]\right)$ $=F^{*}\left(\left[\rho_{n}\right]\right), C$ and $D$ are simply the ranges of $\hat{F}$ and $F^{*}$ respectively, and (7) is equivalent to $\hat{F}$ being one-to-one while (8) is equivalent to $F^{*}$ being one-to-one.

9.4. Notations and assumptions as in 9.3. The following conditions are equivalent to $F$ being one-to-one: (1)-(6) as in 8.3;

(7) $F^{*}$ is onto $(F / X)$;

(8) $C=$ Bor $/ X$;

(9) $D=F / X$.

Proof. (6) $\Longleftrightarrow(7)$ has been noted above. Since $C=\operatorname{range}(\hat{F}), D=$ range $\left(F^{*}\right),(8)$ says that $\hat{F}$ is onto and (9) says that $F^{*}$ is onto.

REMARK. The conditions concerning $C$ and $D$ were added to show that these results say something about the behavior of sequences $\left\langle\left[\varphi_{n}\right]_{X}\right| n\langle\omega\rangle$ of elements of Bor $/ X$ in the B.a. $F / X$, without having to talk about functions (thus (2) $\Longleftrightarrow(7) \Longleftrightarrow(8)$ in 9.3 and (2) $\Longleftrightarrow(8) \Longleftrightarrow(9)$ in 9.4).

We are now ready for the main result of this section, which shows just how large $F / X$ is for any analytic set $X$.

9.5. THEOREM. Let $X$ be an analytic set.

(a) If $X$ is countable, $F / X$ is isomorphic to $P(X)$ (the B.a. of all subsets of $X)$.

(b) If $X$ is uncountable and Borel, $F / X \cong F$.

(c) If $X$ is non-Borel, then $F / X$ and $F$ are not isomorphic, but each of them has a < $<$-complete embedding in the other and a $<k$-complete homomorphism onto the other.

Proof. (a) If $X$ is countable, $X=\operatorname{Mod}(\sigma)$, then by 6.1(a) $\sigma$ is strongly confining and so, as the proof of 5.1 shows, each $\varphi$ supported by $\omega$ is equivalent modulo $\sigma$ to the countable B.t. $\bigvee_{x \in \operatorname{Mod}(\varphi)^{s d}(x) \text {. Thus }}$ $\left(\forall \varphi \in B T^{<k}(\omega)\right)\left(\exists \chi \in B T^{\omega}\right)\left([\varphi]_{X}=[\chi]_{X}\right)$; hence $F / X=$ Bor $/ X$. But Bor $/ X$ is simply $P(X)$ when $X$ is countable hence the assertion. 
(b) If $X$ is uncountable and Borel then by $7.3 X$ and $\omega_{2}$ are isomorphic in the Borel category $\mathbf{B}$; hence also in $\mathbf{A}$. As a functor, ${ }^{*}$ preserves isomorphisms, and therefore $F / X$ and $F / \omega_{2}=F$ are isomorphic in $A^{*}$; hence isomorphic as B.a's.

(c) $X$ is analytic but not Borel. By 7.4 there exist one-to-one and onto analytic mappings $X \rightarrow \omega_{2}, \omega_{2} \rightarrow X$. Applying * to these four mappings and using 9.3, 9.4 we get the desired homomorphisms in (c). It remains to show that $F$ and $F / X$ are not isomorphic. If they are, let $f: F \rightarrow F / X, g: F / X \rightarrow$ $F$ be an isomorphism and its inverse. Note that $F=F / \omega_{2}=F / \Lambda \varnothing$. Choose a code $\sigma$ for $X$ as an analytic set, and let $(\sigma, \bar{\varphi}, \wedge \varnothing)$ be a code for $f$ (as an $\mathbf{A}^{*}$-morphism) and $(\Lambda \varnothing, \bar{\psi}, \sigma)$-a code for $g$.

The facts that $g \circ f=1_{F}$ and $f \circ g=1_{F / X}$ can be expressed by:

$$
R_{(\bigwedge \varnothing, \bar{\psi}, \sigma)}^{*} \circ R_{\left(\sigma, \bar{\varphi}, \wedge_{\phi}\right)}^{*}=R_{\left(\Lambda \phi,\left(p_{n} \mid n<\omega\right), \wedge \phi\right)}^{*}
$$

and

$$
R_{(\sigma, \bar{\varphi}, \wedge \phi)}^{*} \circ R_{(\bigwedge \phi, \bar{\psi}, \sigma)}^{*}=R_{\left.\left(\sigma,\left\langle p_{n}\right| n<\omega\right), \sigma\right)}^{*}
$$

By the formal rules of calculation developed above this is equivalent to: $\varphi_{n}(\bar{\psi}) \equiv$ $p_{n}$ and $\left(\psi_{n}(\bar{\varphi}) \equiv p_{n}\right)(\sigma)$ for all $n$. Now, $\sigma \in H C$, so if $\bar{\varphi}, \bar{\psi}$ satisfying $\varphi_{n}(\bar{\psi}) \equiv p_{n},\left(\psi_{n}(\bar{\varphi}) \equiv p_{n}\right)(\sigma)$ for all $n$ exist at all, such $\bar{\varphi}, \bar{\psi}$ can be found, by Lévy's theorem, in $H C$. But this means that $f$ and $g$ can be chosen as

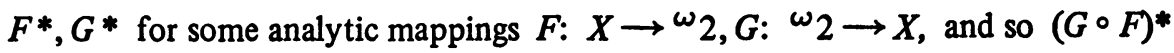
$=f \circ g=1_{F / X}=\left(1_{X}\right)^{*}$ and similarly $(F \circ G)^{*}=\left(1_{\left(\omega_{2}\right)}\right)^{*}$, but $*$ is oneto-one; hence $G \circ F=1_{X}$ and $F \circ G=1\left(\omega_{2}\right)$, contradicting the fact (7.4) that $X$ and $\omega_{2}$ are not isomorphic in $\mathbf{A}$. This completes the proof.

REMARK. 1. The proof of (b) actually shows that if $X$ and $Y$ are any two analytic sets isomorphic in $A$ then the B.a's $F / X$ and $F / Y$ are isomorphic.

2. We know that $|F|=2^{k} \geqslant k$ (the fact that $|F| \geqslant k$ follows easily by considering the elements $\left[\pi_{\alpha}\right], \alpha<\kappa$ of $F$, where $\pi_{\alpha}$ are the nonequivalent B.t's of §1). 9.5 shows that if $X$ is analytic and uncountable, then $|F| X|=| F \mid$.

We shall now apply 9.5 to direct factors of $F$ determined by Borel sets. Our terminology and notation are these: For any B.a. $B$ and element $b$ of $B$, the direct factor of $B$ determined by $b$ is a B.a. $B_{1}=\{x \in B \mid x \leqslant b\}$ with the partial ordering inherited from $B$. We denote this direct factor by $b \wedge B$, in order to remind us that $\langle b \wedge x \mid x \in B\rangle$ is a complete homomorphism of $B$ onto $b \wedge B$.

Note that for any $\varphi \in H(\kappa)$ the function given by $[\chi]_{\varphi} \mapsto[\varphi \wedge \chi]$ $(\chi \in H(\kappa))$ is an isomorphism between $B T^{<\kappa}(\omega) / \varphi \vdash$ and the direct factor of $F$ determined by $[\varphi]$. In particular, when $\varphi \in B T^{\omega}, F / \varphi \cong[\varphi] \wedge F$, but $[\varphi]$ 
is identified with the Borel set $\operatorname{Mod}(\varphi)$, so from 9.5(a), (b) we get

9.6. Let $B$ be a Borel set. If $|B| \leqslant \aleph_{0}$ then the direct factor of $F$ determined by $B(B \wedge F)$ is isomorphic to $P(B)$, and if $|B|>\aleph_{0}$ then $B \wedge$ $F$ is isomorphic to $F$.

REMARK. In order to get only 9.6 , or $9.5(a)$, (b), we could restrict ourselves throughout to B.t's supported by $\omega$ and to Borel (rather than analytic) sets. Had we done so, 9.1 and 9.2 could be proved without using Lévy's theorem (for instance in 9.1 we would have $\sigma_{1} \equiv \sigma_{2}$ ). Then the functor * could be introduced as we have done. 9.3 and 9.4 would still require this theorem, but they have not been used in the proof of 9.5(a), (b). Thus $9.5(\mathrm{a}),(\mathrm{b})$ and 9.6 are obtained without Lévy's theorem, and this gives (using the fact that $|F| \rightarrow \infty$ when $\kappa \rightarrow \infty$ ) an alternative proof that if $\sigma \in B T^{\omega},|\operatorname{Mod}(\sigma)|>\aleph_{0}$ then $\sigma$ is nonconfining on $\omega$.

10. Automorphisms of $F$. We ask which properties (or relations, functions etc.) of Borel sets can be expressed as algebraic properties of them as elements of the free B.a. F. For example, being countable is such a property, because for $B \in$ Bor, $|B|$ is just the number of atoms in $F$ below $B$.

The question is not very precise, but at least we can say that we are interested in properties (relations etc.) that are invariant under automorphisms of $F$. Therefore let us introduce the following terminology: Let $B$ be a B.a., $a, b \in$ B. $a$ and $b$ are said to be similar in $B\left(a \cong \cong^{B} b\right)$ when $b=f(a)$ for some automorphism $f$ of $B$. Similarly, if $a_{i}, b_{i} \in B$ for $i \in I$, the families $\bar{a}=$ $\left\langle a_{i} \mid i \in I\right\rangle, \bar{b}=\left\langle b_{i} \mid i \in I\right\rangle$ are said to be similar in $B(\bar{a} \cong \bar{b} \bar{b})$ when there is an automorphism $f$ of $B$ such that $f\left(a_{i}\right)=b_{i}$ for each $i \in I$.

We can now pose a more precise problem: Let $\bar{A}=\left\langle A_{i} \mid i \in I\right\rangle, \bar{B}=$ $\left\langle B_{i} \mid i \in I\right\rangle$ be two families of Borel sets. Find a necessary and sufficient condition that $\bar{A} \cong \mathrm{F} \bar{B}$.

Similarity in Bor can be decided as follows:

10.1. Lemma. Let $\bar{A}, \bar{B}$ be as above. $\bar{A} \cong \cong^{\mathrm{Bor}} \bar{B}$ iff there is a Borelautomorphism $F$ of $\omega_{2}$ such that $B_{i}=F^{-1}\left(A_{i}\right)$ for each $i \in I$.

["Borel-automorphism" means an automorphism in the category B, i.e., a measurable permutation having a measurable inverse; however, the inverse is automatically measurable by 7.1.]

Proof. Automorphisms of Bor $=B$ Bor $/ \omega_{2}$ are just the functions $\hat{F}$ corresponding to Borel-automorphisms $F$ of $\omega_{2}$, because the categories $B$ and $\widehat{B}$ are anti-isomorphic by the functor ${ }^{\wedge}$. Thus $\bar{A} \cong{ }^{\text {Bor }} \bar{B}$ iff there is a Borel automorphism $F$ of $\omega_{2}$ such that $\hat{F}\left(A_{i}\right)=B_{i}$, i.e., $B_{i}=F^{-1}\left(A_{i}\right)$, for each i. Q.E.D. 
For finite sequences of Borel sets $\bar{A}=\left\langle A_{i}\right| i\langle n\rangle, \bar{B}=\left\langle B_{i}\right| i\langle n\rangle$, it is easy to see from 7.3 that the condition in the lemma is satisfied iff for each sequence $\left\langle\epsilon_{i} \mid i<n\right\rangle \in{ }^{n} 2,\left|\bigcap_{i<n} \epsilon_{i} \cdot A_{i}\right|=\left|\bigcap_{i<n} \epsilon_{i} \cdot B_{i}\right|$ (where $1 \cdot X=X$, $0 \cdot X=\sim X=\omega_{2} \sim X$ for any $X \subseteq \omega_{2}$ ), because then the $2^{n}$ Borel-isomorphisms $F_{\epsilon}: \bigcap_{i} \epsilon_{i} \cdot B_{i} \rightarrow \bigcap_{i} \epsilon_{i} \cdot A_{i}(\epsilon: n \rightarrow 2)$ can be combined to a Borel automorphism $F$ of $\omega_{2}$ such that $F^{-1}\left(A_{i}\right)=B_{i}$ for $i<n$.

For infinite sequences of Borel sets I do know such a simple characterization of similarity in Bor.

What about similarity in F? It follows from $\$ 9$ that every automorphism $\hat{F}$ of Bor has a unique extension to an automorphism $F^{*}$ of $F$. Therefore, any two families that are similar in Bor are similar in $F$. One consequence of this is

10.2. Theorem. Let $A_{i}, B_{i}(i<n)$ be Borel sets. Then $\left\langle A_{i}\right| i\langle n\rangle$ $\cong{ }^{\mathrm{F}}\left\langle B_{i}\right| i\langle n\rangle$ iff $\left\langle A_{i}\right| i\langle n\rangle \cong{ }^{\mathrm{Bor}}\left\langle B_{i}\right| i\langle n\rangle$ iff for each $\epsilon: n \rightarrow 2$, $\left|\bigcap_{i<n} \epsilon_{i} \cdot A_{i}\right|=\left|\bigcap_{i<n} \epsilon_{i} \cdot B_{i}\right|$. In particular, if $A, B$ are Borel sets then $A \cong F$ $B$ iff $|A|=|B|,|\sim A|=|\sim B|$.

Proof. We have seen above that if the condition on cardinalities holds then the sequences are similar in Bor, hence in $F$. The condition is necessary because $\left|\bigcap_{i<n} \epsilon_{i} \cdot A_{i}\right|$ is the number of atoms in $F$ (or Bor) below $\bigcap_{i<n} \epsilon_{i}$ - $A_{i}$ and is thus preserved by automorphisms of $F$ (or of Bor).

The theorem just proved shows that for a finite number of Borel sets, the only properties that can have an algebraic meaning in $F$ are those determined by the cardinalities of possible intersection of these sets and their complements.

We conclude with the following reduction of similarity in $F$ to similarity in Bor.

10.3. Lemma. Let $\bar{A}=\left\langle A_{i} \mid i \in I\right\rangle, \bar{B}=\left\langle B_{i} \mid i \in I\right\rangle$ be families of Borel sets. If $I$ is countable then $\bar{A} \cong{ }^{F} \bar{B} \Longleftrightarrow \bar{A} \cong{ }^{\text {Bor }} \bar{B}$ (as noted above, $\Leftarrow$ holds for any $I$ ).

Proof. It suffices to prove $\Rightarrow$ for $I=\omega$. For each $n<\omega$ choose codes $\sigma_{n}, \rho_{n}$ for $A_{n}, B_{n}$ respectively as Borel sets $\left(\sigma_{n}, \rho_{n} \in B T^{\omega}\right)$ and let $\bar{\sigma}=$ $\left\langle\sigma_{n}\right| n\langle\omega\rangle, \bar{\rho}=\left\langle\rho_{n}\right| n\langle\omega\rangle(\bar{\sigma}, \bar{\rho} \in H C)$. The rest of the proof is similar to the proof of 9.5(c). One shows that the automorphism $f$ of $F$ taking $A_{n}$ to $B_{n}$ for each $n$ and its inverse $g=f^{-1}$ can be coded by sequences $\bar{\varphi}, \bar{\psi} \in H(k)$ satisfying some syntactical conditions $\left(\varphi_{n}(\bar{\psi}) \equiv p_{n} \equiv \psi_{n}(\bar{\varphi}), \sigma_{n}(\bar{\varphi}) \equiv \rho_{n}\right.$ for each $n$ ), and concludes by Lévy's theorem that if such $\bar{\varphi}, \bar{\psi}$ exist they can be chosen in $H C$, so that the automorphisms $f, g$ have the form $F^{*}, G^{*}$ for some Borelautomorphisms $F, G$, and then $\hat{F}$ is an automorphism of Bor taking $A_{n}$ to $B_{n}$ for each $n$. Thus if $\bar{A}$ and $\bar{B}$ are similar in $F$ they are similar in Bor too. 
Here is an example which shows that some restriction on $I$ in 10.3 is essential. In the example we suppose that $\kappa>2^{\aleph_{0}}$. Let $K$ be a nonmeasurable permutation of $\omega_{2}, \bar{A}=\left\langle A_{i} \mid i \in I\right\rangle, \bar{B}=\left\langle B_{i} \mid i \in I\right\rangle$ where $I=\omega_{2}$ and for each $i, A_{i}=\{K(i)\}, B_{i}=\{i\}$. There is no Borel-automorphism $F$ of $\omega_{2}$ such that $B_{i}=F^{-1}\left(A_{i}\right)$ for each $i$, because this implies $F=K$. Thus $\bar{A} \not \#^{\mathrm{B} o r} \bar{B}$. However, consider for each $n$ the B.t.s $\varphi_{n}, \psi_{n}$ defined as follows $(s d(x)=$ $\bigwedge_{n} x_{n} \cdot p_{n}$ for each $x \in \omega_{2}$, and $\left.P_{n}=\{x \mid x(n)=1\}\right)$ :

$$
\begin{aligned}
& \varphi_{n}=\left[\left(\neg \underset{x \in \omega_{2}}{\vee} s d(x)\right) \wedge p_{n}\right] \vee \vee\left\{s d(x) \mid x \in \omega_{2}, K(x) \in P_{n}\right\} ; \\
& \psi_{n}=\left[\left(\neg \underset{x \in \omega_{2}}{\vee} s d(x)\right) \wedge p_{n}\right] \vee \vee\left\{s d(x) \mid x \in \omega_{2}, K^{-1}(x) \in P_{n}\right\} .
\end{aligned}
$$

Let $\bar{\varphi}=\left\langle\varphi_{n}\right| n\langle\omega\rangle, \bar{\psi}=\left\langle\psi_{n}\right| n\langle\omega\rangle$. If $\left.\kappa\right\rangle 2^{\kappa_{0}}$ then $\bar{\varphi}, \bar{\psi} \in H(\kappa)$. And then $R_{(\bigwedge \phi, \bar{\varphi}, \bigwedge \phi)}^{*}$ and $R_{(\bigwedge \phi, \bar{\psi}, \bigwedge \phi)}^{*}$ are an automorphism of $F$ and its inverse, and the former takes $A_{i}$ to $B_{i}$ for each $i$ To see this one must show that for each $n<\omega, x \in \omega_{2:} \varphi_{n}(\bar{\psi}) \equiv p_{n} \equiv \psi_{n}(\bar{\varphi})$ and $(s d(K(x)))(\bar{\varphi}) \equiv s d(x)$ (because $\left.A_{x}=\operatorname{Mod}(\operatorname{sd}(K(x))), B_{x}=\operatorname{Mod}(s d(x))\right)$. This is verified by a routine computation, which is facilitated by proving the equivalences separately modulo $\chi$ and modulo $\neg \chi$ where $\chi=\bigvee_{x \in \omega_{2}} s d(x)$.

$[\bar{\varphi}$ and $\bar{\psi}$ above can be arrived at by imagining a larger universe in which the set $I=\left(\omega_{2}\right.$ of "our" universe $)$ is countable, and there considering the Borel functions $F$ and $G$, defined on $\omega_{2}$ of that universe, which coincide with $K$ and $K^{-1}$ respectively on $I$ and with the identity function on $\sim I . \bar{\varphi}$ and $\bar{\psi}$ are simply codes for these Borel functions.]

11. Concluding remarks and applications. It would be nice to know that any two analytic non-Borel sets are isomorphic in A. This would imply, as in 9.5(b), that if $X$ and $Y$ are two analytic non-Borel sets then $F / X \cong F / Y$. Independently of whether the assertion above is true or false, there is the interesting question of characterizing the B.a's Bor $/ X$ (or $F / X$ ) for analytic nonBorel sets $\boldsymbol{X}$ in algebraic terms (like the characterization of Bor as a free $\sigma$ B.a. on $\aleph_{0}$ generators) and classifying them if they are not all isomorphic.

Another problem of descriptive set theory is to replace the condition for similarity in Bor in 10.1 by a more useful one (cf. the condition in 10.2), at least for $\omega$-sequences.

We now wish to indicate how the coding of sets and functions by B.t's in $\S 8$ can be utilized besides its role in making possible the applications of Lévy's theorem in $\S \S 9-10$ and besides its possible role in a constructive development of descriptive set theory, about which we know as yet very little. 
The work on $\S 8$ seems useful in the domain of absoluteness results for properties of Borel and analytic sets and functions (given by their codes) between transitive models of set theory. Such results (under a different coding) for Borel sets play a basic role in Solovay's work about Lebesgue measurability in various models, and it is clear that his work [So] can be continued by a general study of parts of analysis in such models, with applications to "ordinary" analysis as well (see [FN] and [We] for such applications). Therefore efficient methods for coding and then proving absoluteness of properties of various objects considered in analysis, like measurable functions, will probably be helpful.

Our coding of Borel sets and functions in $\S 8$ is indeed very convenient to work with, and the absoluteness of such properties of Borel sets as being empty, finite, countable, closed, perfect is shown for this coding by verifying, for instance, that the formulas " $\varphi \in B T^{\omega}$ and $\operatorname{Mod}(\varphi)$ is closed", " $\varphi \in B T^{\omega}$ and $\operatorname{Mod}(\varphi)$ is not closed" are both $\Sigma_{1}^{Z F C}$ (or $\Sigma_{1}^{Z F}$ if necessary). The $\Delta_{1}$-ness of "L" and " $\cong$ " is the key to many of these results. Also by simple combinations of the various equivalent conditions mentioned in $\S 8$ for functions or mappings one can show the absoluteness of the assertions that a Borel mapping (as given by a code $(\sigma, \bar{\varphi}, \rho))$ is one-to-one or that it is an isomorphism in the category $\mathbf{B}$. Another useful point is that operations on Borel functions like composition can be represented very simply on the codes.

Let us stress, however, that some properties of Borel, and especially analytic, objects are nonabsolute under our coding, or any other coding satisfying some natural requirements. Let us give a very rough outline of an example. We cannot "decide", for $\sigma \in H C$, whether $\operatorname{Mod}(\sigma)=\omega_{2}$ or not because $Q=\{\sigma \in H C \mid$ $\operatorname{Mod}(\sigma)=\omega_{2}$ is $\Pi_{1}$ - but not $\Sigma_{1}$-definable over $H C$. In fact, we can show that if $P$ is any $\Pi_{2}^{1}$ subset of $\omega_{2}$ then there is a function $F \Delta_{1}$-definable over $H C$ such that $\left(\forall x \in \omega_{2}\right)[x \in P \Longleftrightarrow F(x) \in Q]$. Thus, if $Q$ were $\Sigma_{1}$ over $H C$ (or $\Sigma_{1}$, i.e., $\Sigma_{1}$ in some parameter) $P$ would also be, and from this it can be inferred that $P$ is a $\Sigma_{2}^{1}$ subset of $\omega_{2}$, which is false for a suitable choice of $P$. Thus $Q$ is not $\Sigma_{1}$ over $H C$ and we can also show (using a nonabsolute number-theoretic $\Sigma_{2}^{1}$ predicate) that (in a certain relative consistency sense) there exist transitive ZFC models $M \subseteq N$ and some $\sigma \in H C^{(M)}$ such that $" \operatorname{Mod}(\sigma)=\omega_{2} "$ holds in $M$ but not in $N$.

APPENDIX. BOOLEAN TERMS, VAlUATIONS AND DERIVATIONS (A FORMAL SUMmARY). The sets $p_{x}=(0, x)$ are called atomic B.t's (or variables). For each $y$ let $\neg y=(1, y), \wedge y=(2, y)$ and $\bigvee y=(3, y)$. By recursion on $\epsilon$ we define:

Definition 1. $x$ is a B.t. iff $x$ is an atomic B.t. or $x=7 y$ for some 
B.t. $y$ or $x=\wedge_{y}$ or $x=\bigvee y$ for some set $y$ of B.t's.

We let $\varphi, \chi, \psi, \cdots \quad$ vary on B.t's and put $\varphi \wedge \psi=\wedge\{\varphi, \psi\}, \varphi \vee \psi=$ $\bigvee\{\varphi, \psi\}, \varphi \rightarrow \psi=7 \varphi \vee \psi, \varphi \leftrightarrow \psi=(\varphi \rightarrow \psi) \wedge(\psi \rightarrow \varphi)$

Next we define by recursion on $\varphi$ the set of subterms $\operatorname{Sub}(\varphi)$.

Definition 2. $\operatorname{Sub}(\varphi)=\{\varphi\}$ if $\varphi$ is atomic; $\operatorname{Sub}(\varphi)=\{\varphi\} \cup \operatorname{Sub}(\psi)$ if $\varphi=\neg \psi ; \operatorname{Sub}(\varphi)=\{\varphi\} \cup \cup_{\psi \in X} \operatorname{Sub}(\psi)$ if $\varphi=\wedge X$ or $\varphi=\bigvee X$.

$\varphi$ is finite when $\operatorname{Sub}(\varphi)$ is finite.

Definition 3. The support of $\varphi$ : $\operatorname{Supp}(\varphi)=\left\{x \mid p_{x} \in \operatorname{Sub}(\varphi)\right\}$.

$\varphi$ is supported by $A$ when $\operatorname{Supp}(\varphi) \subseteq A$.

Definition 4. Substitution: Let $f$ be a function whose values are B.t's. Define $\varphi(f)$ by recursion of $\varphi$ as follows:

$$
\begin{aligned}
& \varphi(f)=f(x) \quad \text { if } \varphi=p_{x}, x \in \operatorname{dom}(f) ; \\
& \varphi(f)=\varphi \quad \text { if } \varphi=p_{x}, x \notin \operatorname{dom}(f) ; \\
& \varphi(f)=\neg \psi(f) \quad \text { if } \varphi=\neg \psi ; \\
& \varphi(f)=\bigwedge_{\psi \in X} \psi(f) \quad \text { if } \varphi=\wedge X, \text { dually for } \vee X .
\end{aligned}
$$

Definition 5. Depth: For any set $A$ of ordinals we let $\operatorname{Sup} A=$ $\sup \{\alpha+1 \mid \alpha \in A\}$. Now the depth $d(\varphi)$ is defined by recusion on $\varphi$ :

$$
\begin{aligned}
& d(\varphi)=0 \quad \text { if } \varphi \text { is atomic; } \\
& d(\varphi)=d(\psi)+1 \quad \text { if } \varphi=7 \psi ; \\
& d(\varphi)=\operatorname{Sup}\{d(\psi) \mid \psi \in X\} \quad \text { if } \varphi=\wedge X \text { or } \varphi=\bigvee X .
\end{aligned}
$$

Definition 6. Let $(\varphi \leqslant \psi)=(4, \varphi, \psi)$. Sets of the form $\varphi \leqslant \psi$ are called inequalities (between B.t's).

Definition 7. An inference is a triple $(k, P, c)$ where $0 \leqslant k \leqslant 8, P$ is a set of inequalities (the premises) and $c$ is an inequality (the conclusion) and one of the following holds [the rules $1-8$ correspond to the definition of a B.a. as a complemented distributive lattice]:

0 . $k=0, P=\varnothing$ (thus $c$ is taken as a nonlogical axiom);

1. $k=1, P=\varnothing, c=(\varphi \leqslant \varphi)$ for some $\varphi$;

2. $k=2$ and $\exists \varphi, \chi, \psi[P=\{\varphi \leqslant \chi, \chi \leqslant \psi\}, c=(\varphi \leqslant \psi)]$;

3. $k=3, P=\varnothing, \exists \varphi X[\varphi \in X, c=(\bigwedge X \leqslant \varphi)]$;

4. $k=4, \exists \varphi X[c=(\varphi \leqslant \wedge X), P=\{\varphi \leqslant \psi \mid \psi \in X\}]$;

5. $k=5, p=\varnothing, \exists \varphi X[\varphi \in X, c=(\varphi \leqslant \bigvee X)]$;

6. $k=6, \exists \varphi X[c=(\bigvee X \leqslant \varphi), P=\{\psi \leqslant \varphi \mid \psi \in X]$;

7. $k=7, P=\varnothing, \exists \varphi \chi \psi[c=(\varphi \wedge(\chi \vee \psi) \leqslant(\varphi \wedge \chi) \vee(\varphi \wedge \psi))]$; 
8. $k=8, P=\varnothing, \exists \varphi \psi[c=((\varphi \wedge \neg \varphi) \leqslant \psi)$ or $c=(\psi \leqslant(\varphi \vee \neg \varphi))]$.

Definition 8. Definition by recursion of $n l$-derivations (i.e., derivations using arbitrary nonlogical axioms): $D$ is a $n l$-derivation iff $D$ has the form $(I, J)$ where $I=(k, P, c)$ is an inference (we call $I$ the last inference of $D$ and $c$ the conclusion of $D$ ) and $J$ is a set of $n l$-derivations such that $P=\{d \mid d$ is the conclusion of some $j \in J$ \} (i.e., in $J$ just the premises of $I$ are derived).

Definition 9. The set of subderivations of a $n l$-derivation $D$ is defined recursively by $\operatorname{Sub}(D)=\{D\} \cup \bigcup_{j \in J} \operatorname{Sub}(j)$ where $D=(I, J)$. The last inferences of subderivations of $D$ are called the inferences of $D$.

Definition 10. Let $\Gamma$ be a set of inequalities, $c$ an inequality. $A$ derivation of $c$ from $\Gamma$ is a nl-derivation $D$ such that $c$ is the conclusion of $D$ and whenever $(0, \not, r)$ is an inference of $D, r \in \Gamma$.

When $\Gamma=\varnothing$ we call $D$ a derivation of $c$.

Definition 11. Let $\Gamma, c$ be as in Definition 10. $\Gamma+c$ when there is a derivation of $c$ from $\Gamma$. $t_{c}$ when $s i t c$.

Definition 12. $\varphi \equiv \psi(\Gamma)$ when $\Gamma \vdash \varphi \leqslant \psi$ and $\Gamma \vdash \psi \leqslant \varphi . \varphi \equiv \psi$ when $\varphi \equiv \psi(\varnothing)$.

Among the elementary properties of the notions introduced thus far we shall mention two.

Lemma 1. Let $\Gamma$ be a set of inequalities, $\chi=\bigwedge\left\{\chi_{1} \rightarrow \chi_{2} \mid\left(\chi_{1} \leqslant \chi_{2}\right)\right.$ $\in \Gamma\}$, and $\varphi, \psi$ B.t's.

(a) $\Gamma \vdash \varphi \leqslant \psi$ iff $\Gamma \vdash \wedge \varnothing \leqslant \varphi \rightarrow \psi$;

(b) $\Gamma \vdash \varphi \leqslant \psi$ iff $\vdash x \wedge \varphi \leqslant \psi$.

Lemma 2 (R ule of Substitution). Let $f$ be as in Definition 4, and extend the substitution operation in the obvious way to inequalities. Let $\Gamma, \varphi$, $\psi$ be as in Lemma 1 and $\Gamma(f)=\{r(f) \mid r \in \Gamma\}$. Then

(a) $\Gamma \vdash \varphi \leqslant \psi \Rightarrow \Gamma(f) \vdash \varphi(f) \leqslant \psi(f)$;

(b) $\varphi \equiv \psi(\Gamma) \Rightarrow[\varphi(f) \equiv \psi(f)](\Gamma(f))$.

Definition 13. A valuation is a pair $(B, I)$ where $B$ is a $B$.a. and $I$ is a function into $B$.

Definition 14. Let $(B, D)$ be a valuation. We define the value $\|\varphi\|=$ $\|\varphi\|_{B, I}$ by recursion on the B.t. $\varphi$ denoting $*=B$ (so that $* \notin B$ ):

$$
\begin{array}{ll}
\|\varphi\|=I(x) & \text { if } \varphi=P_{x}, x \in \operatorname{dom}(I) ; \\
\|\varphi\|=\neg^{B}\|\psi\| & \text { if } \varphi=\neg \psi,\|\psi\| \in B ; \\
\|\varphi\|=\wedge^{B} A & \text { if } \varphi=\wedge X, A=\{\|\psi\| \psi \in X\}, A \subseteq B \text { and } A \text { has a } \\
& \text { meet in } B ; \text { dually for } \bigvee X ;
\end{array}
$$


$\|\varphi\|=* \quad$ in any other case.

Definition 15. The B.t. $\varphi$ is defined in the valuation $(B, D)$ when $\|\varphi\|_{B, I} \in B$ (i.e. $\neq * *$ ). The inequality $(\varphi \leqslant \psi)$ is defined in $(B, I)$ when $\varphi$ and $\psi$ are both defined in $(B, D) .(\varphi \leqslant \psi)$ is true (false) in $(B, D)$ when it is defined and $\|\varphi\|_{B, I} \leqslant\|\psi\|_{B, I}\left(\|\varphi\|_{B, I} \leqslant\|\psi\|_{B, I}\right.$ resp.).

Definition 16. A two-valued model is a function $I$ into 2 . We often identify $I$ either with the valuation $(\overline{2}, I)$, where $\overline{2}$ is the B.a. $\{0 \leqslant 1\}$, or with the pair $(A, B)$ when $A=\operatorname{dom}(I)$ and $B=\{x \in A \mid I(x)=1\}$. When $\|\varphi\|_{\overline{2}, I}=1(=0)$ we say that $\varphi$.is true (false resp.) in $I$.

When $\varphi$ is true in $I$ we write also $I \mid=\varphi$ (thus $p_{x}$ is defined in $(A, B)$ (where $B \subseteq A$ ) iff $x \in A$, true iff $x \in B$ and false iff $x \in A \sim B$, identifying $(A, B)$ with the corresponding $I)$.

Definition 17. Semantical analogues of $\vdash, \equiv$ : Let $\Gamma$ be a set of inequalities, $\varphi$ and $\psi$ B.t's, $c=(\varphi \leqslant \psi) . \Gamma \vdash^{1} c(c$ is a Boolean consequence of $\Gamma$ ) when $c$ is true in every valuation in which each element of $\Gamma$ is true and $c$ is defined; $\varphi \equiv^{1} \psi(\Gamma)$ when $\Gamma \vdash^{1} \varphi \leqslant \psi$ and $\Gamma \vdash^{1} \psi \leqslant \varphi$ (i.e., $\|\varphi\|=\|\psi\|$ in every valuation in which each element of $\Gamma$ is true and $\varphi, \psi$ are defined). When $\Gamma=\varnothing$ we write $\vdash^{1} c, \varphi \equiv^{\mathbf{1}} \psi$.

If we restrict the valuations in this definition to valuations in $\overline{2}$ we get the definitions of the corresponding two-valued concepts: $\Gamma \vdash^{2} c, \varphi \equiv^{2} \psi(\Gamma), \vdash^{2} c$, $\varphi \equiv^{2} \psi$.

Definition 18. Algebras of B.t's (Lindenbaum algebras): Let $T$ be a nonempty set of B.t's closed under $\neg, \wedge, \vee$ and subterms, and let $\Gamma$ be a set of inequalities. $T / \Gamma \vdash$ is the B.a. whose members are the equivalence classes $[\varphi]=\{\psi \in T \mid \varphi \equiv \psi(\Gamma)\}$ for $\varphi \in T$, and in which $[\varphi] \leqslant[\psi]$ iff $\Gamma \vdash \varphi \leqslant$ $\psi$. (Existence and uniqueness of such a B.a. are easily proved.)

Lemma 3. Let $T, \Gamma$ and $[\varphi]$ for $\varphi \in T$ be as in Definition 18 and $B=T / \Gamma \vdash$. Then for all $\varphi \in T$ and all $\psi, X$ :

$\varphi=\neg \psi \Rightarrow \psi \in T,[\varphi]=\urcorner^{B}[\psi]$;

$\varphi=\bigwedge X \Rightarrow X \subseteq T,[\varphi]=\wedge_{\psi \in X}[\psi]$, and dually for $\bigvee X$.

Lemma 4. Let $T, \Gamma,[\varphi](\varphi \in T)$ be as in Definition 18. Put $B=T / \Gamma \vdash$, $I=\left\langle\left[p_{x}\right]\right| x$ is such that $\left.P_{x} \in T\right\rangle$. Then for any $\varphi \in T, \varphi$ is defined in $(B$, I) and $\|\varphi\|_{B, I}=[\varphi]$.

Using this lemma (for one direction) one proves the

Completeness theorem. Let $\Gamma, \varphi, \psi, c$ be as in Definition 17. Then $\Gamma \vdash c \Longleftrightarrow \Gamma \vdash^{1} c ; \varphi \equiv \psi(\Gamma) \Longleftrightarrow \varphi \equiv^{1} \psi(\Gamma)$.

REMARK. This is a theorem of ZF (in fact of much weaker theories). The 
notions defined in Definitions 1-10,13-16 are clearly $\Delta_{1}^{Z F}$ (in fact they are primitive recursive in the sense of [JK]). Therefore $\Gamma \vdash c, \varphi \equiv \psi(\Gamma)$ are $\Sigma_{1}^{Z F}$ formulas (asserting the existence of derivations), and $\Gamma H^{1} c, \varphi \neq^{1} \psi(\Gamma), \Gamma H^{2} c$, $\varphi \neq^{2} \psi(\Gamma)$ are $\Sigma_{1}^{Z F}$ formulas (asserting the existence of valuations in which certain inequalities are true and others false). Note the importance of defining valuations without requiring the B.a. to be complete in ensuring $\Sigma_{1}^{Z F}$-ness of $H^{1}$.

It follows from these remarks and the completeness theorem that ${ }_{,}, \equiv$ are $\Delta_{1}^{Z F}$. An alternative proof using Barwise's completeness theorem is to note that

$\Gamma \forall c \Longleftrightarrow$ there is an admissible set $A$ such that $\Gamma, c \in A$ and there is no derivation of $c$ from $\Gamma$ in $A$.

\section{REFERENCES}

[Ba] J. Barwise, Absolute logics and $L_{\infty} \omega$, Ann. Math. Logic 4 (1972), 309-340.

[FN] J. E. Fenstad and D. Normann, On absolutely measurable sets, Fund. Math. 81 (1974), 91-98.

[Ga] H. Gaifman, Infinite Boolean polynomials. I, Fund. Math. 54 (1964), 229-250. MR 29 \#5765.

[Gr] J. Gregory, Incompleteness of a formal system for infinitary finite-quantifler formulas, J. Symbolic Logic 36 (1971), 445-455.

[JK] R. B. Jensen and C. Karp, Primitive recursive set functions, Proc. Sympos. Pure Math., vol. 13, part I, Amer. Math. Soc., Providence, R. I., 1971, 143-167. MR 43 \#7317.

[Ka] C. Karp, A proof of the relative consistency of the continuum hypothesis, Sets, Models and Recursion Theory (Proc. Summer School Math. Logic and Tenth Logic Colloq., Leicester, 1965), North-Holland, Amsterdam, 1967, 1-32. MR 36 \#1320.

[Lé] A. Lévy, A hierarchy of formulas in set theory, Mem. Amer. Math. Soc. No. 57 (1965). MR 32 \#7399.

[Na] M. Nadel, An application of set theory to model theory, Israel J. Math. 11 (1972), 386-393. MR 46 \#3298.

[Pa] K. R. Parthasarathy, Probability measures on metric spaces, Probability and Mathematical Statistics, no. 3, Academic Press, New York, 1967. MR 37 \#2271.

[So] R. M. Solovay, A model of set theory in which every set of reals is Lebesgue measurable, Ann. of Math. (2) 92 (1970), 1-56. MR 42 \#64. appear).

[St] J. Stavi, Extensions of Kripke's embedding theorem, Ann. Math. Logic (to

[We] E. Wesley, Extensions of the measurable choice theorem by means of forcing, Israel J. Math. 14 (1973), 104-114.

INSTITUTE OF MATHEMATICS, THE HEBREW UNIVERSITY OF JERUSALEM, JERUSALEM, ISRAEL

Current address: Department of Mathematics, Stanford University, Stanford, California 94305 